\title{
SCDT: Detecting somatic CNVs of low chimeric ratio in cf-DNA
}

Zhaoyang Qian ${ }^{1,2,3, \dagger, \text { Xiaofeng Wang }}{ }^{1,2,3,}$, Chang Shi ${ }^{1,2,3}$, Rui Han ${ }^{4}$, Yaoshen Wang $^{4}$, Hongmei $\mathrm{Zhu}^{1,2,3, *}$

1. BGI-Shenzhen, Shenzhen 518083, China

2. China National GeneBank, BGI-Shenzhen, Shenzhen 518120, China

3. Binhai Genomics Institute, BGI-Tianjin, BGI-Shenzhen, Tianjin 300308, China

4. BGI Genomics, BGI-Shenzhen, Shenzhen 518083, China

*To whom correspondence should be addressed

$\dagger$ The authors wish it to be known that, in their opinion, the first two authors should be regarded as Joint First Authors.

\begin{abstract}
Motivation: Sequencing of cell-free DNA (cf-DNA) has enabled Noninvasive Prenatal Testing (NIPT) and"liquid biopsy" of cancers. However, while the aneuploidy and point mutations were focused on by most of NITP and liquid biopsy studies, detecting sub-chromosome CNVs that affect a few to dozens of megabases was rarely reported, likely attributable to the difficulty in accurately identifying them, especially for those present in a small fraction of cf-DNA.

Results: We developed a somatic CNV detection tool (SCDT), for detecting sub-chromosome CNVs in cf-DNA using whole genome sequencing (WGS) data or off-target reads in target sequencing data. Additional to using control samples for correcting genome position specific bias, two GC correction steps were performed,
\end{abstract}


which regressed GC content of DNA fragments and that of genome bins, respectively. After GC correction, the coefficients of variation of copy ratios approximated the lower boundary of theoretical values, suggesting removing of almost all systematic errors. Finally, CNVs were detected by a piecewise least squares fitting based segmentation algorithm, which outperformed other segmentation methods. We applied SCDT on simulated and real maternal plasma samples, and target cf-DNA sequencing of 118 normal individuals and 240 cancer patients, and demonstrated high sensitivity and specificity.

$\begin{array}{llll}\text { Availability: } & \text { SCDT } & \text { available }\end{array}$ https://github.com/Martiantian/Somatic_cnv_detect_tool.

Contact: zhuhongmei@genomics.cn

Supplementary Information: Supplementary data are available at Bioinformatics online

\section{Introduction}

Copy number variation $(\mathrm{CNV})$ is one type of structural variation with duplication or deletion event that affects a considerable number of base pairs (Sharp, et al., 2005), altering gene dosage and subsequently affecting functional and biological behavior of cells. CNVs have been known to greatly contribute to a wide repertoire of human diseases, including genetic disease, developmental and neuropsychiatric disorders (Kirov, et al., 2009; Sebat, et al., 2007; Walsh, et al., 2008) and almost all types of cancers (Pollack, et al., 2002; Shlien and Malkin, 2009; Taylor, et al., 2008). 
Detecting CNVs in human genomes has been a routine clinical test for disease screening, diagnosis and therapy guiding.

In the recent years, plasma cell free DNA (cf-DNA) sequencing has been broadly applied to non-invasive genetic diagnostics. One of the most important applications of cf-DNA sequencing is non-invasive prenatal testing (NIPT), which directly sequences cf-DNA extracted from maternal blood to identify likely aneuploidy of the fetus. However, NIPT generally focused on whole-chromosome aneuploidies (triploid 13/18/21, X, XXY and XYY) which account for only $30 \%$ of all live births with a chromosome abnormality. Recent progression has been made in genome-wide screening of sub-chromosomal CNVs with significantly smaller sizes, which have a considerably higher incidence $(0.5-1.7 \%)$ than whole-chromosome aneuploidy in human pregnancy (Brady, et al., 2016), and could be associated with genetic disease including DiGeorge syndrome (22q11.2 deletion), Cri-du-chat syndrome (5p deletion), Angelman syndrome (15q11-q13 deletion) and 1p36 deletion syndrome. However, non-invasively detecting CNVs with small chimeric fraction without previously known positions is much more challenging than aneuploidy testing. After two proof-of-concept studies (Jensen, et al., 2012; Peters, et al., 2011) on a few cases, several subsequent studies focused on achieving statistical significance using relatively high depth whole genome sequencing (more than 100 million reads). These methods were based on statistical test on individual genomic bins, or required several consecutive bins to be significant (Srinivasan, et al., 2013; Yu, et al., 2013) However, in addition to the cost of relatively deep whole genome sequencing, high rates of false 
positives (FPs) and false negatives (FNs) in these individual-bin test methods would restrict their application in real clinical use. Some other methods reduced the requirement on sequencing depth by employing sliding window strategy (Straver, et al., 2014) or using binary segmentation with dynamic threshold (Chen, et al., 2013), and aimed at detecting large fragment aberrations (>10M) using low-coverage sequencing data. Lo et al, reported $60.7 \%$ (17/28) of accuracy for analyzing $3 \mathrm{Mb}$ to $42 \mathrm{Mb}$ de novo $\mathrm{CNVs}$ using 4-10 M reads, while the sensitivity increased to $92.9 \%$ using relative higher sequencing depth up to 120M reads (Lo, et al., 2016). Yin et al developed a method to identify 69 of 73 (94.5\%) CNVs identified by array CGH using 10 million reads, with a specificity of 98.1\% (Yin, et al., 2015). A method based on unified Hidden Markov model was developed for detecting fetal CNVs and achieved great resolution $(400 \mathrm{~kb})$ with fetal fraction of $13 \%$, however, is only feasible using deep sequencing data and information of parental SNP genotypes, which is unavailable in routine NIPT (Rampasek, et al., 2014).

Another inspiring application of cf-DNA sequencing is to be used as a surrogate for tissue biopsy, named"liquid biopsy”, for screening and monitoring tumor-derived genomic aberrations. Circulating tumor DNA (ct-DNA) can be detected in the plasma of cancer patients, and has great potential in clinical management of cancers. A plenty of targeted therapies have been developed to target copy number change of some cancer driver genes, e.g. high level amplifications of ERBB2, MET, CCND1 and FGFR1 (Baselga and Swain, 2009; Christensen, et al., 2005; Musgrove, et al., 2011; Turner, et al., 2010), etc. However, current reported noninvasive assays seldom 
include detection of actionable CNVs, which may be due to the serious difficulty on accurately identifying CNVs in ct-DNA, as ct-DNA typically accounts for a little proportion $(<10 \%)$ of cf-DNA, even in many advanced stage cancer patients(Adalsteinsson, et al., 2017). Most published studies employed reads counting strategy for genome bins and simple statistics such as Z-test to identify CNVs. Chan et al used individual bin based Z-test on 4 high depth whole genome sequencing (WGS) (17X) of HCC cases (Chan, et al., 2013). Heitzer et al calculated segment z-score after CNV segmentation using circular binary segmentation (CBS) algorithm in 13 plasma samples of 9 metastatic prostate patients, which usually have high tumor DNA concentrations (Heitzer, et al., 2013). Xu et al performed individual bin z-score based CNV analysis on 31 patients, and showed that recognizable CNVs were only detectable in most samples with large tumor size (tumor dimension $>50 \mathrm{~mm})(\mathrm{Xu}$, et al., 2015). However, method accurately determining the CNV fragment using shallow depth WGS data with low FPs is scarce and tools for identifying tumor-derived CNV in cf-DNA of a wide range of patients would have significant clinical values.

In this study, we present a novel approach named Somatic CNV Detecting Tool (SCDT), which has ability to use shallow WGS data and target sequencing data to detect genome-wide microdeletions or microduplications (MDs) without a priori knowledge of an event's location. To maximize the ability to remove "noise" introduced by library construction, PCR process and sequencing, and intrinsic difference between genome regions, we used control samples to correct genome position specific bias, and two GC correction steps to regress GC content of DNA 
fragments and that of genome bins, respectively. Following that we nearly achieve the theoretical minimum of random fluctuation in copy ratios. A segmentation algorithm based on piecewise least squares fitting and a rigorous statistical method is applied to finally determine the CNVs. We show that SCDT recovered all "spiking in" MDs with $\geq 3 \mathrm{Mbp}$ length and $\geq 5 \%$ chimeric fraction $(\geq 10 \%$ mix ratio) using about 25 million sequencing reads $(0.3 \times$ genome coverage). Finally, we applied our algorithm in three cf-DNA datasets of abnormal maternal plasma samples, normal samples and a large cohort of patients with various types of cancer, respectively, and demonstrated the feasibility of SCDT for precisely detecting clinically relevant CNVs in cf-DNA.

\section{Methods}

\subsection{Data preparation and Overview of methods}

We use bam format files as input of SCDT, including at least one sample as control.

The control samples should be prepared using the same protocol with the test samples, including methods for library constructing and sequencing. Duplicate reads should be removed in the bam files using software such as Picard-tools and Samtools. The aligned genome positions of reads were extracted from the bam files, with a filtering step to discard reads with low mapping quality or high number of mismatches. Additionally, for using target sequencing data as input, reads located adjacent to ( $<500 \mathrm{bp})$ the target region were discarded from the bam files.

Firstly, the whole genome should be divided into non-overlapping bins (defined as level-1 bins) with fixed length assigned by users. Read depth count (RDC) of each 
level-1 bin is obtained by counting reads with start positions in it. However, each DNA fragment was not counted by 1 , but instead by 1 divided by a correction factor corresponding to its CG content (section 2.2). Secondly, the RDCs of each test and control sample were centralized to 1 by dividing their medians, and then we used the mean of centralized RDCs at each bin across the control samples to generate a reference data, which is used to normalize the centralized RDCs of test samples to obtain the copy ratios (section 2.3). Thirdly, we merged a fixed number (defined by users) of level-1bins into the level-2 bin. The copy ratio of each level-2 bin was calculated as the mean of copy ratios of level-1 bins inside it. Subsequently, we performed a second step of GC correction based on regression for the copy ratios and GC content of level-2 bins using general liner model (GLM) (section 2.4). Finally, we performed CNV segmentation by a piecewise least squares fitting on the copy ratios of level-2 bins (section 2.5), and tested the significance of each CNV segment under the assumption of independent and identical distribution of copy ratios (section 2.6) (Supplementary Fig S1).

\subsection{First step of GC correction based on single DNA fragment}

Firstly, we divided the GC-content range (0-1) into 1000 intervals (0.001 per interval), followed by calculating GC-content distribution on the $1000 \mathrm{GC}$ intervals for 170 base-pair (bp) sliding windows (sliding with $1 \mathrm{bp}$ each time) in the reference genome (except the sex chromosomes). Secondly, for single-end sequencing data, we extended the sequencing reads to $170 \mathrm{bp}$ based on its alignment position in the reference 
genome. Then we calculated GC-content distribution of extended reads on the 1000

GC intervals for each sample. GC-content intervals higher than $70 \%$ and lower than $20 \%$ were discarded for their intense fluctuation of read counts. For each DNA fragment, a correction factor $c f$ was assigned by:

$$
c f_{g c^{i}}=n_{g c^{i}} / n_{g c^{i}}^{0}
$$

where $g c^{i}$ is GC interval of the DNA fragment $\mathrm{i}, n_{g c^{i}}^{0}$ is distribution density of $g c^{i}$ in the reference genome, $n_{g c^{i}}$ is distribution density of $g c^{i}$ in sequenced DNA fragments of this sample (Fig 1A). So we can get the normalized read depth count (NRDC) of each level-1 bin by

$$
n r d c_{j}=\sum_{i \in b i n_{j}} 1 / c f_{g c^{i}}
$$

in which i represents the ID of reads whose start position located in the jth bin.

\subsection{Control RDC construction and copy ratio calculation}

To reduce systematic bias caused by factors other than GC-content, we used the copy ratio of test sample NRDC to the control reference NRDC (CNRDC) for further analysis. CNRDC is calculated by the following procedures: Firstly, to eliminate the influence of variation in sequencing data volumes, all the samples including test samples and control samples should be performed with centralization of NRDC by dividing the median NRDC of all genome bins. Secondly, to reduce the random fluctuation in the CNRDC and thus reduce the fluctuation in the final copy ratio, we construct CNRDC by averaging the NRDCs at each bin across the control samples.

After obtaining the CNRDC, we can get the corrected copy ratio (CCR) for the test 
samples by

$$
c c r_{j}=\frac{n r c d_{j}}{c n r d c_{j}}
$$

in which $c c r_{j}$ is the corrected copy ratio of the jth bin, $n r d c_{j}$ is the NRDC of the jth bin in the test sample, and $c n r d c_{j}$ is the CNRDC of the jth bin. Additionally, to avoid the frequent germline CNVs, outlying bins with CNRDC $<m_{C N R D C} * 0.7$ or CNRDC $>m_{C N R D C} * 1.4$ were removed, in which $m_{C N R D C}$ is the median value of CNRDC of all autosomal bins.

For detecting CNVs at various level of length, we performed CNV segmentation on detection bins (defined as level-2 bins), whose length (should be an integer multiple of level-1 bin length) are assigned by users. The copy ratio of each level-2 bin was obtained by averaging CCR of all level-1 bins within it.

\subsection{Second step of GC-correction}

Even with the first step of GC correction described in 2.2, we observed that the CCRs were still generally correlated with GC-content (Supplementary Fig S2). The remaining bias, though slightly increasing the variance of CCR, may produce false positives in several GC abnormal regions in the genome, e.g. chr1p and chr19. Thus a second step of GC-correction was performed to further remove the remaining GC bias, by a generalized linear regression for CCR and GC-content of level-2 bins, which can be illustrated as:

$$
\begin{aligned}
& c c r_{j}=a * g c_{j}^{2}+b^{*} g c_{j}+c+e \\
& c c r_{j}^{\prime}=e_{j}
\end{aligned}
$$


in which $a, b$ and $c$ are coefficients of regression, $g c_{j}$ is $\mathrm{GC}$-content of $\mathrm{jth}$ level-2 bin, $c c r_{j}^{\prime}$ is the GC-corrected copy ratio of jth level-2 bin, and $e_{j}$ is the residual of $c c r_{j}$. Then, $c c r_{j}^{\prime}$ was used instead of $c c r_{j}$ as input for $\mathrm{CNV}$ segmentation algorithm.

\subsection{CNV Segmentation}

To locate the breakpoints of CNVs and determine the CNV status of segments, we employed a piecewise least squares estimation model based on stepwise regression for each chromosome, which could also be described as piecewise linear fitting of ladder type and identified the breakpoints of ladders one by one. We minimized sum of squares of residuals to get the least squares estimation, as described below.

If we have obtained the sorted breakpoint set

$$
E=\left\{(0,1),\left(j_{2}, j_{2}+1\right),\left(j_{3}, j_{3}+1\right), \ldots \ldots,\left(j_{k}, j_{k}+1\right),(n, n+1)\right\} \quad\left(1<j_{2}<j_{3}<\ldots<j_{k}<n,\right.
$$

each breakpoint is indicated by two consecutive genomic bins and $\mathrm{n}$ is the total bin numbers of this chromosome) after fitting $\mathrm{k}$ ladders to a chromosome, we can obtain the estimation of $c c r_{j}$ for each bin:

$$
\hat{c c r} r_{j}=\left\{\begin{array}{cc}
\overline{c c r}_{1, j_{2}} & j=1, \cdots, j_{2} \\
\vdots & \vdots \\
\overline{c c r}_{j_{k-1}+1, j_{k}} & j=j_{k-1}+1, \cdots, j_{k} \\
\overline{c c r}_{j_{k}+1, n} & j=j_{k}+1, \cdots, n
\end{array}\right.
$$

in which $\overline{c c r}_{j_{k-1}+1, j_{k}}=1 /\left(j_{k}-j_{k-1}\right) \sum_{j=j_{k-1}+1}^{j_{k}} c c r_{j}$, or the mean value of $c c r_{j}$ from $\left(j_{k-1}+1\right)$ th bin to $j_{k}$ th bin. So the sum of squares of residuals is

$$
E S S_{k}=\sum_{j=1}^{n}\left(c c r_{j}-\overline{c c r}_{j}\right)^{2}
$$


Next, we introduce the detailed steps of the piecewise linear fitting model by taking the chromosome $\mathrm{i}$ for example. For the $\mathrm{jth}$ bin of chromosome $\mathrm{i}$, let $D_{i}=\left\{z_{j}^{i} \mid z_{j}^{i}=c c r_{j}^{i}-c c r_{j}^{i}+1, j=1,2, \cdots, n_{i}\right\}$ be the discriminant set for chromosome $\mathrm{i}$, in which $n_{i}$ is the total bin number.

Step 0: Get the initial value of coefficient of variation (CV). We firstly calculated CV of each 20 consecutive level-2 bins in all autosomes by

$$
C V=S_{C C R} / \overline{C C R}
$$

where $S_{C C R}$ and $\overline{C C R}$ are the standard variation and the average of CCRs, respectively. . Then the initial $\mathrm{CV}\left(c v_{0}\right)$ could be estimated by averaging the smallest $30 \% \mathrm{CVs}$ of all 20 consecutive level-2 bins in the whole genome, and subsequently be used in the loop termination conditions for the piecewise linear fitting. When $\mathrm{k}=1$, the breakpoint set is $E_{k}^{i}=\left\{(0,1),\left(n_{i}, n_{i}+1\right)\right\}$, which has only two breakpoints and one CNV segment. The estimation of $\hat{c c r}{ }_{j}^{i}$ could be calculated by the mean of all $c c r_{j}^{i}$ in chromosome $i$.

Step 1: Judge whether to terminate the loop of piecewise linear fitting for chromosome i. For discriminant set $D_{i}$, we can calculate the $\mathrm{CV}$ value of ladder $\mathrm{k}$ $\left(c v_{k}\right)$ by

$$
c v_{k}=s_{i} / \bar{z}_{i},
$$

in which $\bar{z}_{i}=1 / n_{i} \sum_{j=1}^{n_{i}} z_{j}^{i}$ is the mean value of $D_{i}$ set, $s_{i}=\sqrt{1 / n_{i} \sum_{j=1}^{n_{i}}\left(z_{j}^{i}-\bar{z}_{i}\right)^{2}}$ is the standard variation of $D_{i}$ set. When $\mathrm{k}=1$, if $c v_{k}-\gamma * c v_{0} \leq 0(\gamma$ is a coefficient assigned by users, and $\gamma=1$ by default) we terminate the loop for piecewise linear fitting. And when k>1, if 


$$
\begin{gathered}
\left(c v_{k-1}^{2}-c v_{k}^{2}\right)(n-1)<(r / 2)^{2} * L / B \\
\text { or } c v_{k}-\gamma * c v_{0} \leq 0
\end{gathered}
$$

we terminate the loop, where $r$ is a coefficient assigned by users corresponding to the lowest chimeric fraction for CNV detecting (3\% by default), B is the length of level-2 bin (1M bp by default) and $\mathrm{L}$ is the smallest size for CNV detecting ( $3 \times \mathrm{b}$, by default). Otherwise, $\mathrm{k}=\mathrm{k}+1$, and then go to step 2 .

Step 2: Add one optimal point to breakpoint set $E_{k-1}^{i}$ by traverse the potential new breakpoint set $R_{k-1}^{i}$. After adding one more breakpoint $j \in R_{k-1}^{i}$ to $E_{k-1}^{i}$, we can get the new breakpoint set $E_{k, j}^{i}$ and the new sum of squares of residuals $\operatorname{ESS}_{k, j}^{i}$. To get the optimal breakpoints for fitting the chromosome, we minimize the $\operatorname{ESS}_{k, j}^{i}$ over all $j \in R_{k-1}^{i}:$

$$
\begin{gathered}
j=\underset{j \in R_{k-1}^{i}}{\arg \min } E S S_{k, j}^{i} \\
\text { and } E^{i} S_{k}^{i}=E S S_{k, j}^{i}
\end{gathered}
$$

Step 3: Test whether the new breakpoint set is significant for fitting chromosome i. According to the stepwise regression model, if $\mathbf{k}>2$, we should test whether including each one of the previous breakpoints in $E_{k-1}^{i}$ is more significant for fitting chromosome $\mathrm{i}$ than including the new breakpoint in step 2. If including a previous breakpoint is not more significant than including the new breakpoint, this previous breakpoint should be removed from the breakpoint set while $\mathrm{k}=\mathrm{k}-1$, until all the remained breakpoints are more significant than the new breakpoint. Then, go to step 1 . The significance of including a breakpoint is assessed by calculating the decrease in 
ESS.

\subsection{Significance test}

For chromosome $\mathrm{i}$ of the test sample, we assume that $c c r_{j}^{i}=c \hat{c r}_{j}^{i}+\varepsilon_{j}^{i}\left(j=1, \cdots, n_{i}\right)$ and $\varepsilon_{j}^{i}$ follow independent identical normal distribution $N\left(0, \sigma_{i}^{2}\right)$. The unbiased estimation of $\hat{\sigma}_{i}$ can be calculated by

$$
\hat{\sigma}_{i}=\sqrt{\frac{1}{n_{i}-1} \sum_{j=1}^{n_{i}}\left(\varepsilon_{j}^{i}-\bar{\varepsilon}_{i}\right)^{2}}, \bar{\varepsilon}_{i}=\frac{1}{n_{i}} \sum_{j=1}^{n_{i}} \varepsilon_{j}^{i} .
$$

So the CCR in a normal bin should follow distribution of $N\left(1, \hat{\sigma}_{i}^{2}\right)$. Therefore, we can inferred that average CCR of the kth segment $\left(\overline{C C R}_{k}\right)$ follows distribution of $N\left(1, \frac{\hat{\sigma}_{i}^{2}}{j_{k+1}-j_{k}}\right)$ under the H0 hypothesis of $\overline{C C R}_{k}=1$, and the significance of the kth segment to reject $\mathrm{H} 0$ could be tested.

Because the additional deviation in the copy ratios induced by real CNVs could not be determined before going through the pipeline, the first run of step 2.5 and 2.6 was only used to define normal regions in the whole genome, and thus used to set parameters $c v_{0}$ for the second run of these two steps to obtain the final segmentation results and the significance of each CNV. Before the second run of step 2.5 and 2.6, the copy ratios across the genome should be centralized again using the average copy ratio of normal bins defined by the first run. The initial coefficient of variation $c v_{0}$ used in the step 0 of 2.5 in the second run is also reset by $c v$ of normal bins at the first run. 


\section{Results}

\subsection{Theoretical limitation of CNV detectability in cf-DNA}

The sequenced DNA fragments only took a very small part of the cf-DNA in the circulation, so the process of blood drawing, cf-DNA extracting and library construction could be regarded as random sampling of cf-DNA from an infinite population. Thus the read count examined in a certain genome bin should follow Poisson distribution. To calculate theoretical limitation of $\mathrm{CNV}$ detectability in cf-DNA, we firstly assessed the theoretical random fluctuation of copy ratios. The RDC in the ith bin could approximate to a random variable following Poisson distribution $P\left(\lambda_{i}\right)$ with a mathematical expectation of $\lambda_{i}$. However, $\lambda_{i}$ may be different for different i because some inherent characteristics in different genomic regions could affect the read count, such as the mappability (Ha, et al., 2012). We have:

$$
\lambda_{i}=P_{i}^{*} N / b
$$

in which $\mathrm{N}$ is the total effective reads of this sample, $\mathrm{b}$ is the total number of level-2 bins in the whole genome, and $P_{i}$ is a position specific coefficient to adjust the reads count in i th bin.

Considering $\mathrm{X}$ and $\mathrm{Y}$ are independent, the random fluctuation of copy ratios could be evaluated by

$$
\begin{aligned}
\operatorname{Var}\left(\frac{X / \lambda_{1}}{Y / \lambda_{2}}\right)= & \operatorname{Var}\left(X / \lambda_{1}\right) \operatorname{Var}\left(\lambda_{2} / Y\right)+\operatorname{Var}\left(X / \lambda_{1}\right) E^{2}\left(\lambda_{2} / Y\right)+\operatorname{Var}\left(\lambda_{2} / Y\right) E^{2}\left(X / \lambda_{1}\right) \\
& >\operatorname{Va}\left(X / \lambda_{1}\right) E^{2}\left(\lambda_{2} / Y\right)
\end{aligned}
$$

in which $\lambda_{1}$ is mathematical expectation for test sample $\mathrm{X}$ and $\lambda_{2}$ is mathematical 
expectation for the control reference Y.

It is easy to deduce a lower bound of CV

$$
C V_{\left(\frac{X / \lambda_{1}}{Y / \lambda_{2}}\right)}>\frac{\sqrt{\operatorname{Var}\left(X / \lambda_{1}\right) E^{2}\left(\lambda_{2} / Y\right)}}{E\left(X / \lambda_{1}\right) E\left(\lambda_{2} / Y\right)}=\sqrt{\operatorname{Var}\left(X / \lambda_{1}\right)}=\sqrt{\frac{1}{\lambda_{1}}}
$$

For brevity, we assume that $P_{i}=1$ for all i in each sample, and use the variance of all bins in a sample as the variance of each bin. Then we can get:

$$
\begin{gathered}
\lambda_{i}=N / b \\
C V_{\left(\frac{X / \lambda_{1}}{Y / \lambda_{2}}\right)}>\sqrt{b / N}
\end{gathered}
$$

In order to evaluate the effectiveness of our GC-correction method, we applied this method to 67 normal maternal plasma samples, and compared the actual coefficient of variation $(\mathrm{ACV})$ before and after GC-correction with theoretical lower limit of coefficient of variation (TCV) for the copy ratios (CR). Before GC-correction, we identified various degrees of correlation between CR and GC-content in the 67 samples, implying that different samples were affected by different levels of GC bias, even with identical number of PCR cycles in the library process. We also identified a broad range of correlation between CRs of different samples (Fig 1B), suggesting the non-independence of $\mathrm{CR}$ in different samples. After GC-correction, ACVs were greatly reduced, as well as the linear correlation between GC-content and CRs, and the correlation of CRs between different samples. Moreover, we approximately achieved the theoretical lower limit of TCV after GC-correction (Fig 1C). These results implied that systematic bias in CRs are mostly contributed by GC bias, which 
could be almost completely removed using our GC-correction method. After GC-correction, CR of different samples obeyed the assumption of independence in step 2.6. However, factors other than GC-bias that introduce systematic bias in the RDC should also affect RDC of the control samples, and thus should be normalized in the CR.

We next assessed theoretical limit of detecting power given a confidence coefficient ( $p$-value). A significant CNV segmentation could be modeled as several continuous bins with deviant CRs. Approximately, we assumed that CR of single bin followed an i.i.d normal distribution, thus the detecting power could be assessed by

$$
r / 2 \geq z_{1-\alpha / 2} \cdot A C V / \sqrt{L / B} \geq z_{1-\alpha / 2} \cdot T C V / \sqrt{L / B}
$$

where $r / 2$ is the chimeric fraction of a heterozygous $\mathrm{CNV}, \alpha$ is the confidence level, $z_{1-\alpha / 2}$ is the $1-\alpha / 2$ quantile of standard normal distribution, $\mathrm{L}$ is the length of CNV, and B is the level-2 bin size. Supplementary Figure S3 shows the smallest chimeric fraction of detectable CNV with various lengths and amount of effective reads. Given $\mathrm{N}=25 \mathrm{M}, \mathrm{b}=3000, \mathrm{~B}=1 \mathrm{M}, \mathrm{L}=3 \mathrm{M}$, and $p=10 \mathrm{e}-5$, we could infer that $r / 2$ $=2.80 \%$.

\subsection{Simulation Study and Comparison between segmentation methods}

To evaluate the performance of SCDT, we blended cf-DNA from a healthy female with DNA from tissues of aborted fetuses whose CNVs had been determined by G-banding karyotyping, to simulate cf-DNA from maternal plasma with abnormal fetus. Using this method and tissues of 11 aborted fetuses, we obtained 108 simulated 
samples with various mixture ratios (MR), including 3\%, 5\%, $8 \%, 10 \%, 15 \%$ and 20\%. All the simulated samples were sequenced with 35bp single-end reads on BGIseq 500 platform. After filtering out reads with low mapping quality $(<Q 30)$ or high mismatch numbers, the average amount of effective reads for each sample is averagely 25M (8M- 69M), which is applicable in current NITP test. Considering that the CNVs of aborted fetuses are different from each other, in this experiment we used the median NRDC of all the samples as the control reference for normalization.

We set the level-1 bin size to $100 \mathrm{~kb}$ and the level-2 bin size to $1 \mathrm{M}$, and then used SCDT to analyze these samples. To reduce false positives, we required a CNV segment with $p$-value smaller than $10 \mathrm{e}-5$ ), and with copy ratio $>1.01$ or $<0.99$. Here, we defined a predicted $\mathrm{CNV}$ as a true positive if it overlapped with at least $50 \%$ of a spike-in CNV. Using SCDT we detected all the spike-in CNVs with chimeric fraction $\geq 5 \%$ (mixture ratios $\geq 10 \%$ ) and length $\geq 3 \mathrm{M}$ and had no false positive with length $\geq 3 \mathrm{M}$ after filtering out putative germline CNVs (Fig 2). We then compared our results with the theoretically detection limitation and found that SCDT detected most of theoretically detectable $\mathrm{CNVs}$, though missed some near the line of theoretically limitation (Supplementary Table S1). However, the spike-in fractions evaluated from CRs of CNVs were lower than expected (Supplementary Fig S4), probably because of failure to remove some large fragment DNA during preparation of fetal DNA.

We compared the performance of SCDT on the simulated samples with the state-of-the-art CNV detection methods, including BIC-seq (Xi, et al., 2011), DNAcopy (Venkatraman and Olshen, 2007), Control-FREEC (Boeva, et al., 2012) 
and CNV-seq (Xie and Tammi, 2009). Considering that CBS and BIC-seq didn't have GC correction workflow, these methods were evaluated following preprocessing by our GC-correction method. For each detector, we adjusted the parameters and cutoffs until the results achieved the fewest false positives with sensitivity of $60 \%$ (Supplementary Method). We observed that our GC-normalization step greatly improved performance of CBS and BIC-seq. For the 56 samples with theoretically detectable CNVs of size $\geq 3 \mathrm{M}$, the segmentation method of SCDT had the highest sensitivity (89.29\%), followed by CBS $(82.14 \%)$ and BIC-seq (76.80\%). When considering all the samples with the $\mathrm{CNV}$ size more than $3 \mathrm{M}$, the segmentation method of SCDT also had higher sensitivity (59.77\%) than CBS (52.87\%) and BIC-seq (49.43\%), while the false discovery rates for SCDT, CBS and BIC-seq were around $1.89 \%, 8 \%$ and $0 \%$. We then compared the average sum of length of false positives in each sample at different level of sensitivity, and demonstrated that the segmentation method of SCDT outperformed the other methods (Fig 3).

\subsection{Real Data Analysis3.3.1 Abnormal maternal plasma samples}

To further evaluate the performance of SCDT, we applied it to cf-DNA sequencing data of real clinical samples, including maternal plasma and plasma of cancer patients. 34 maternal plasma samples carring abnormal fetal CNVs previously determined by amniotic fluid puncture and G-banding karyotyping were sequenced with average effective reads of $18 \mathrm{M}(11 \mathrm{M}-31 \mathrm{M})$. We chose another 9 normal maternal plasma samples to construct the control reference. The parameter setting was the same with 
the simulated data described above. We detected all confirmed CNVs in the 34 cases with only one false positive with length $\geq 3 \mathrm{M}$, indicating high sensitivity and specificity of SCDT (Fig 4; Supplementary Table S2). We applied SCDT on another 7 cases of maternal plasma reported with abnormal genotyping by BGI NIPT workflow, but reported negative by amniocentesis and G-banding karyotyping. Interestingly, we observed chimeric CNVs in all these 7 cases, with great significance (Supplementary Fig S5). None of these women had been identified with a cancer, and inconsistence in these cases might result from chimeric placenta, abnormal hematologic clones of maternal, or false negative reports of amniocentesis.

\subsubsection{Target sequencing of cf-DNA for normal individuals}

We then investigated CNVs in 118 cf-DNA target capture sequencing data from normal individuals. All these samples were target enriched using a panel covering 1.7 megabases before sequenced with paired-end 100bp reads on Hiseq2500 platform. To detect CNVs using off-target reads as analogue of low-depth WGS data, we filtered out reads on or near target regions to build whole genome sequencing depth for these plasma samples. The number of off-target reads is $58 \mathrm{M}$ averagely (27-88M). Using $\mathrm{p}<10 \mathrm{e}-5$ for cut-off and filtering out a few frequent false positive regions (totally $27 \mathrm{M}$ ) that are affected by polymorphic CNVs or harbor long centromere and telomere sequence, the false positive callings was evaluated to be $0.03 \%$ of the whole genome (Supplementary Fig S6). However, the false discovery rate should be overestimated, for some of false positives were induced by germline events.

\subsubsection{Target sequencing of cf-DNA for cancer patients}


Finally, the same method for analyzing target-off reads was applied on additional 240 cf-DNA samples of patients with various types of cancers (Supplementary Table S3), which were captured by the same target panel used for normal individuals, with off-target reads of $45 \mathrm{M}$ averagely (16-170M). 10 female samples in the normal dataset were used to build the control reference. Blood cell DNA of these patients was also target sequenced as control to determine somatic point mutations. The most frequent copy number changes in 240 samples included gains of chr1q, 3q, 8q and loss of chr1p, 4, 8p, 17p, consisting with the $\mathrm{CNV}$ profiles in cancers (Fig 5, Supplementary Table S4). We evaluated the concentration of ct-DNA in cf-DNA using both point mutations and CNVs, and identified a high correlation between them ( $r=0.72$, Supplementary Method). Samples detected with CNVs have significantly more point mutations and higher mutational variant allele frequency (VAF, $p<1 \mathrm{e}-10)$ (Fig 5). Of the 107 samples that have $>10 \%$ of the genome detected with CNVs, 97 $(90.7 \%)$ were with average mutational VAF $>2 \%$, while in other 86 cancer samples that have $\leq 1 \%$ of the genome detected with CNVs, , only $6(7.0 \%)$ were with average mutational VAF $>2 \%$ and another $54(62.8 \%)$ sample were absence of point mutations. In addition, we used VAF of point mutations to assess the copy number of CNVs, and detected several targetable agents including high amplification $(\mathrm{CN} \geq 7)$ of EGFR $(n=9), \operatorname{MET}(n=3), \operatorname{ERBB} 2(n=5), \operatorname{KRAS}(n=5)$ FGFR1 $(n=1), \operatorname{CCND} 1(n=7)$, CDK4 $(\mathrm{N}=4)$ et al (Fig 6; Supplementary Fig S7). However, high amplification $(\mathrm{CN} \geq 7)$ of some other genes known to have a role in drug resistance could also be detected in several samples, such as MYC $(n=8)$ and MCL1(n=4), etc. 


\section{Discussion}

Deviation in the copy ratio of each genome bin is composed of at least two components, one is natural fluctuation caused by the random sampling process in blood drawing, DNA extracting, library constructing and sequencing, and the other is systematic fluctuation caused by factors such as GC non-uniform distribution or different success rate of sequencing or reads mapping in certain genome regions. According to the theoretical statistic rules, the natural fluctuation would result in poisson distribution of the final read count per genomic region, and thus can be exactly evaluated. We showed that the limit detection ability for a CNV with certain chimeric fraction and length only depends on the read count per bin, according to the theoretical statistic rules. In this study we introduced a GC correction approach to remove almost all the deviation in copy ratios contributed by non-random factors in cf-DNA sequencing data. However, as FP rate particularly concern clinical application in large scale population screening, and even a low FP rate could produce considerable FP cases, avoiding FPs of biological sources, including germline CNVs, placental mosaicism and maternal abnormality, are noteworthy in addition to reducing FPs of technical source. Several studies have identified CNVs of hemopoietic origin in blood cell-DNA in about 1-3\% of normal individuals (Jacobs, et al., 2012; Laurie, et al., 2012), while they are reasonable to also present in cf-DNA, which is mainly derived from blood cell DNA (Sun, et al., 2015).

Cf-DNA sequencing brought immense opportunities for molecular diagnosis in 
clinical settings, especially in the field of cancer. However, while point mutations and methylation of cf-DNA have been widely used as biomarkers for cancer screening, early detection, treatment guiding, and disease monitoring, the cf-DNA copy-number signatures, another important kind of genomic aberrations and targets of a handful of drugs, is still seldom mentioned and evaluated in studies, partially because of the low ct-DNA fraction and technique difficulty in overcoming the low signal to noise ratio for $\mathrm{CNV}$ detection. Another concern is that quantifying low chimerical CNVs using WGS data requires big data size and increases the cost. However, we demonstrated that using target-off reads, the by-product of target sequencing, was a feasible way to profile somatic CNVs in cf-DNA. In addition to providing information in guiding therapeutic decisions, interrogating cf-DNA CNVs may also help to evaluate the therapy efficiency and monitor tumor recurrence. However, according to our analysis, the detection limit of chimeric ratio of $\mathrm{CNV}$ is inversely proportional to the square of read count, which indicates that 10 folds improvement of sensitivity requires 100 folds of sequencing data, and thus hampers the interrogating of ultra-low chimeric CNVs. Therefore point mutations may be better biomarkers for samples with low ct-DNA fraction. Moreover, as ct-DNA fraction in early stage cancers is extremely low $(0.1 \%$ or less), CNVs in ct-DNA are hard to be profiled and thus not economically applicable to early cancer detection. Overall, integrating CNV analysis into liquid biopsy offers a global view of genomic aberrations, and provides more opportunity for clinical management of cancer.

To accurately find more common chromosomal abnormalities with smaller size at 
earlier gestational stage and cancer stage is an important goal of non-invasive clinical testing. As continuous reduction in sequencing cost, larger data size will become available soon with affordable cost. This allows for more precise diagnostics as our method is expected to perform better with increased sequencing depth. A higher coverage will allow for more stable calls and using smaller bin sizes while keeping the read depth per bin high enough to detect changes confidently.

\section{Acknowledgements and Funding}

The research reported in this work was supported by BGI Academy of Life Sciences and BGI Institute of big data without any official funding.

\section{References}

Adalsteinsson, V.A., et al. (2017) Scalable whole-exome sequencing of cell-free DNA reveals high concordance with metastatic tumors, NAT COMMUN, 8, 1324.

Baselga, J. and Swain, S.M. (2009) Novel anticancer targets: revisiting ERBB2 and discovering ERBB3, NAT REV CANCER, 9, 463-475.

Boeva, V., et al. (2012) Control-FREEC: a tool for assessing copy number and allelic content using next-generation sequencing data, BIOINFORMATICS, 28, 423-425.

Brady, P., et al. (2016) Clinical implementation of NIPT - technical and biological challenges, CLIN GENET, 89, 523-530.

Chan, K.C., et al. (2013) Cancer genome scanning in plasma: detection of tumor-associated copy number aberrations, single-nucleotide variants, and tumoral heterogeneity by massively parallel sequencing, CLIN CHEM, 59, 211-224.

Chen, S., et al. (2013) A method for noninvasive detection of fetal large deletions/duplications by low coverage massively parallel sequencing, Prenat Diagn, 33, 584-590.

Christensen, J.G., et al. (2005) c-Met as a target for human cancer and characterization of inhibitors for therapeutic intervention, CANCER LETT, 225, 1-26. Ha, G., et al. (2012) Integrative analysis of genome-wide loss of heterozygosity and monoallelic expression at nucleotide resolution reveals disrupted pathways in triple-negative breast cancer, GENOME RES, 22, 1995-2007.

Heitzer, E., et al. (2013) Tumor-associated copy number changes in the circulation of 
patients with prostate cancer identified through whole-genome sequencing, GENOME $M E D, \mathbf{5}, 30$.

Jacobs, K.B., et al. (2012) Detectable clonal mosaicism and its relationship to aging and cancer, NAT GENET, 44, 651-658.

Jensen, T.J., et al. (2012) Detection of microdeletion 22q11.2 in a fetus by next-generation sequencing of maternal plasma, CLIN CHEM, 58, 1148-1151.

Kirov, G., et al. (2009) Support for the involvement of large copy number variants in the pathogenesis of schizophrenia, HUM MOL GENET, 18, 1497-1503.

Laurie, C.C., et al. (2012) Detectable clonal mosaicism from birth to old age and its relationship to cancer, NAT GENET, 44, 642-650.

Lo, K.K., et al. (2016) Limited Clinical Utility of Non-invasive Prenatal Testing for Subchromosomal Abnormalities, AM J HUM GENET, 98, 34-44.

Musgrove, E.A., et al. (2011) Cyclin D as a therapeutic target in cancer, NAT REV CANCER, 11, 558-572.

Peters, D., et al. (2011) Noninvasive prenatal diagnosis of a fetal microdeletion syndrome, N Engl J Med, 365, 1847-1848.

Pollack, J.R., et al. (2002) Microarray analysis reveals a major direct role of DNA copy number alteration in the transcriptional program of human breast tumors, Proc Natl Acad Sci U S A, 99, 12963-12968.

Rampasek, L., et al. (2014) Probabilistic method for detecting copy number variation in a fetal genome using maternal plasma sequencing, BIOINFORMATICS, 30, i212-i218.

Sebat, J., et al. (2007) Strong association of de novo copy number mutations with autism, SCIENCE, 316, 445-449.

Sharp, A.J., et al. (2005) Segmental Duplications and Copy-Number Variation in the Human Genome, The American Journal of Human Genetics, 77, 78-88.

Shlien, A. and Malkin, D. (2009) Copy number variations and cancer, GENOME $M E D, \mathbf{1}, 62$.

Srinivasan, A., et al. (2013) Noninvasive detection of fetal subchromosome abnormalities via deep sequencing of maternal plasma, AM J HUM GENET, 92, 167-176.

Straver, R., et al. (2014) WISECONDOR: detection of fetal aberrations from shallow sequencing maternal plasma based on a within-sample comparison scheme, NUCLEIC ACIDS RES, 42, e31.

Sun, K., et al. (2015) Plasma DNA tissue mapping by genome-wide methylation sequencing for noninvasive prenatal, cancer, and transplantation assessments, Proc Natl Acad Sci U S A, 112, E5503-E5512.

Taylor, B.S., et al. (2008) Functional copy-number alterations in cancer, PLOS ONE, 3, e3179.

Turner, N., et al. (2010) FGFR1 amplification drives endocrine therapy resistance and is a therapeutic target in breast cancer, CANCER RES, 70, 2085-2094.

Venkatraman, E.S. and Olshen, A.B. (2007) A faster circular binary segmentation algorithm for the analysis of array CGH data, BIOINFORMATICS, 23, 657-663.

Walsh, T., et al. (2008) Rare structural variants disrupt multiple genes in 
neurodevelopmental pathways in schizophrenia, SCIENCE, 320, 539-543.

$\mathrm{Xi}$, R., et al. (2011) Copy number variation detection in whole-genome sequencing data using the Bayesian information criterion, Proc Natl Acad Sci U S A, 108, E1128-E1136.

Xie, C. and Tammi, M.T. (2009) CNV-seq, a new method to detect copy number variation using high-throughput sequencing, BMC BIOINFORMATICS, 10, 80.

$\mathrm{Xu}, \mathrm{H}$., et al. (2015) Non-invasive Analysis of Genomic Copy Number Variation in Patients with Hepatocellular Carcinoma by Next Generation DNA Sequencing, $J$ CANCER, 6, 247-253.

Yin, A.H., et al. (2015) Noninvasive detection of fetal subchromosomal abnormalities by semiconductor sequencing of maternal plasma DNA, Proc Natl Acad Sci U S A, 112, 14670-14675.

Yu, S.C., et al. (2013) Noninvasive prenatal molecular karyotyping from maternal plasma, PLOS ONE, 8, e60968. 


\section{Figures:}
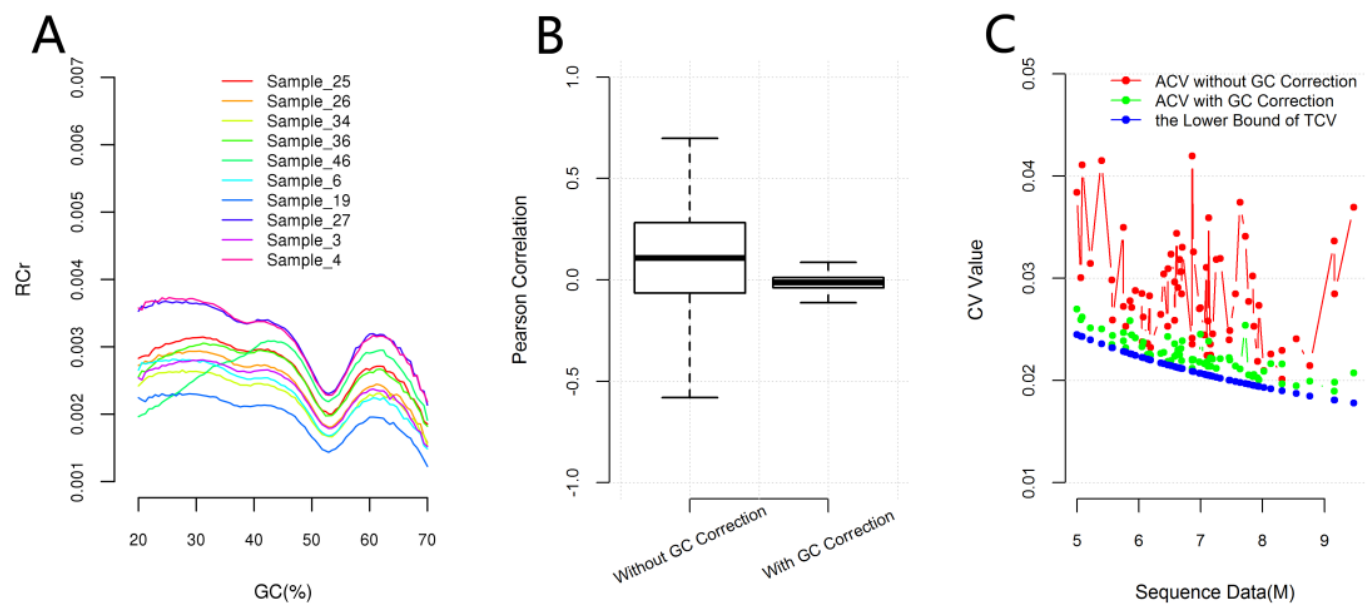

Figure 1. GC correction in 67 normal maternal plasma samples. (A) GC correction factors for DNA fragments based on the GC content by equation (2). This figure only shows ten samples randomly selected from the 67 normal maternal plasma samples. (B) Comparison of Pearson's correlation between copy ratios of different samples before and after GC correction. This figure demonstrates that GC correction significantly $(\mathrm{p}<2.2 \mathrm{e}-16$; Wilcoxon signed rank test with continuity correction using the absolute values of the Pearson's correlation value) reduced the Pearson's correlation between copy ratios of different samples. (C) The theoretical coefficient of variations (TCVs) and the actual coefficient of variations (ACVs) of copy ratios before and after GC correction. The ACVs after GC correction are much closer to the lower bounds of TCVs than the ACVs without GC correction. ACVs after GC correction are only 0.0015(0.00028-0.0057) larger than the lower bounds of TCVs, implying that our GC correction approximately removed all the systematic errors in the copy ratios, including that caused by GC-biases. 

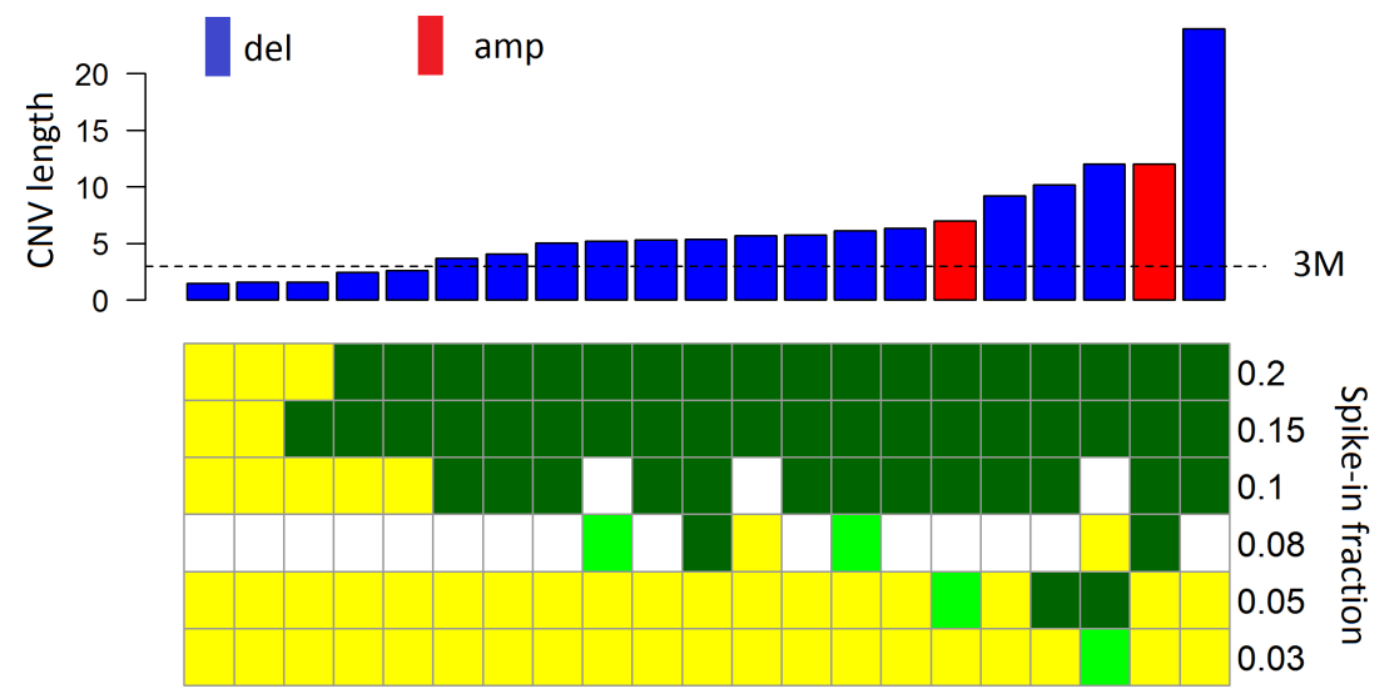

$\mathrm{p}<1 \mathrm{e}-5$

$p<1 e-4$

$\mathrm{p} \geq 1 \mathrm{e}-4$ or undetected

Figure 2. Performance of SCDS on the data of simulated maternal plasma samples. The top panel presents the lengths of spike-in amplifications (red bars) and deletions (blue bars). The lower panel presents the detecting results of spike-in CNVs, while whites blocks denote that these simulations had not been performed. 


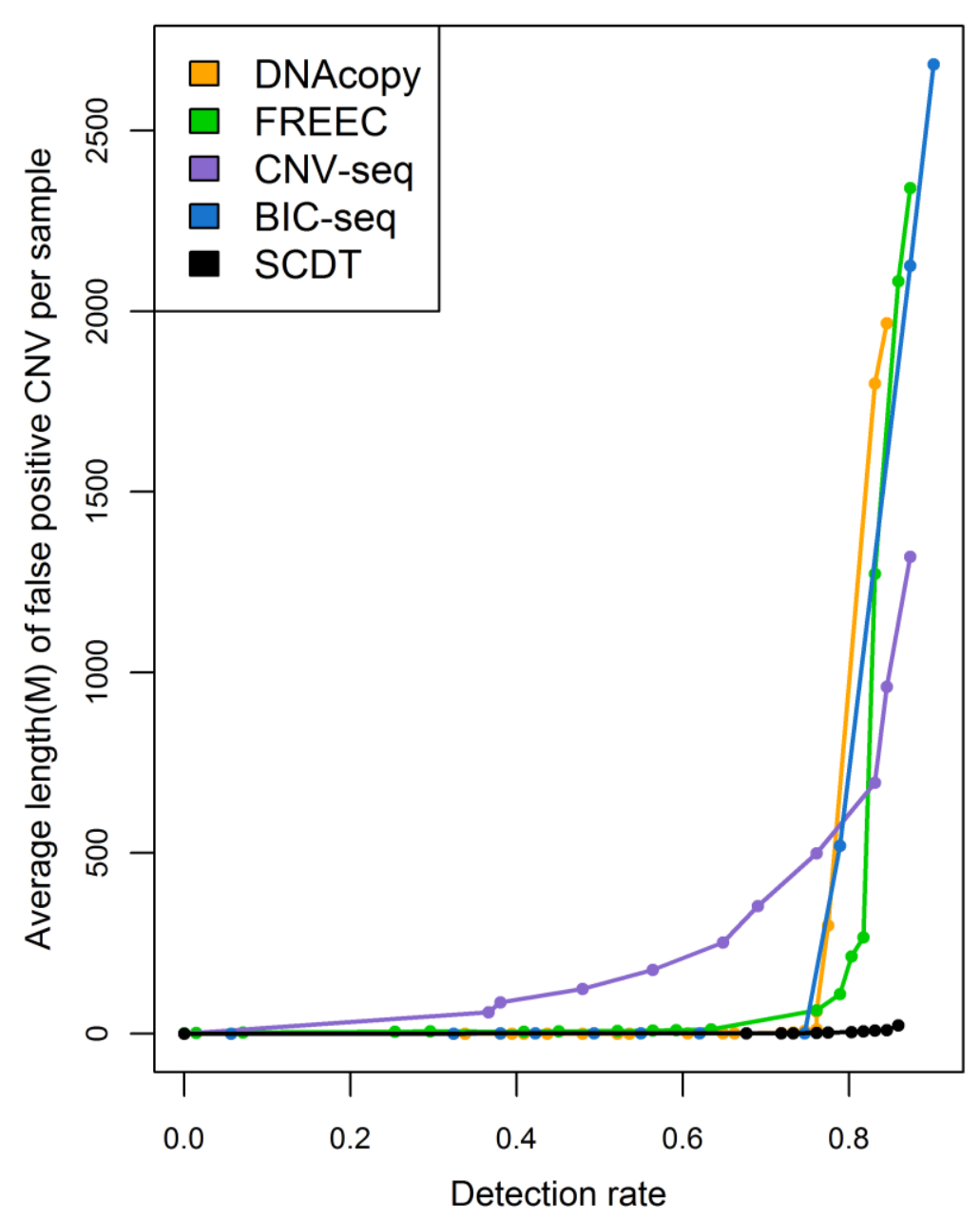

Figure 3. Performance of different methods on the simulated maternal plasma samples. Performances of SCDS and the state-of-the-art CNV detection methods, including BIC-seq (Xi, et al., 2011), DNAcopy (Venkatraman and Olshen, 2007), Control-FREEC (Boeva, et al., 2012) and CNV-seq (Xie and Tammi, 2009), were evaluated by the average length of all false positives in samples with certain sensitivities on the simulated maternal plasma samples (62 samples with $\mathrm{CNV}$ length $\geq 3 \mathrm{M}$ and spike-in fraction $\geq 0.05$ ). Parameter setting of different methods was detailed in the results section. 


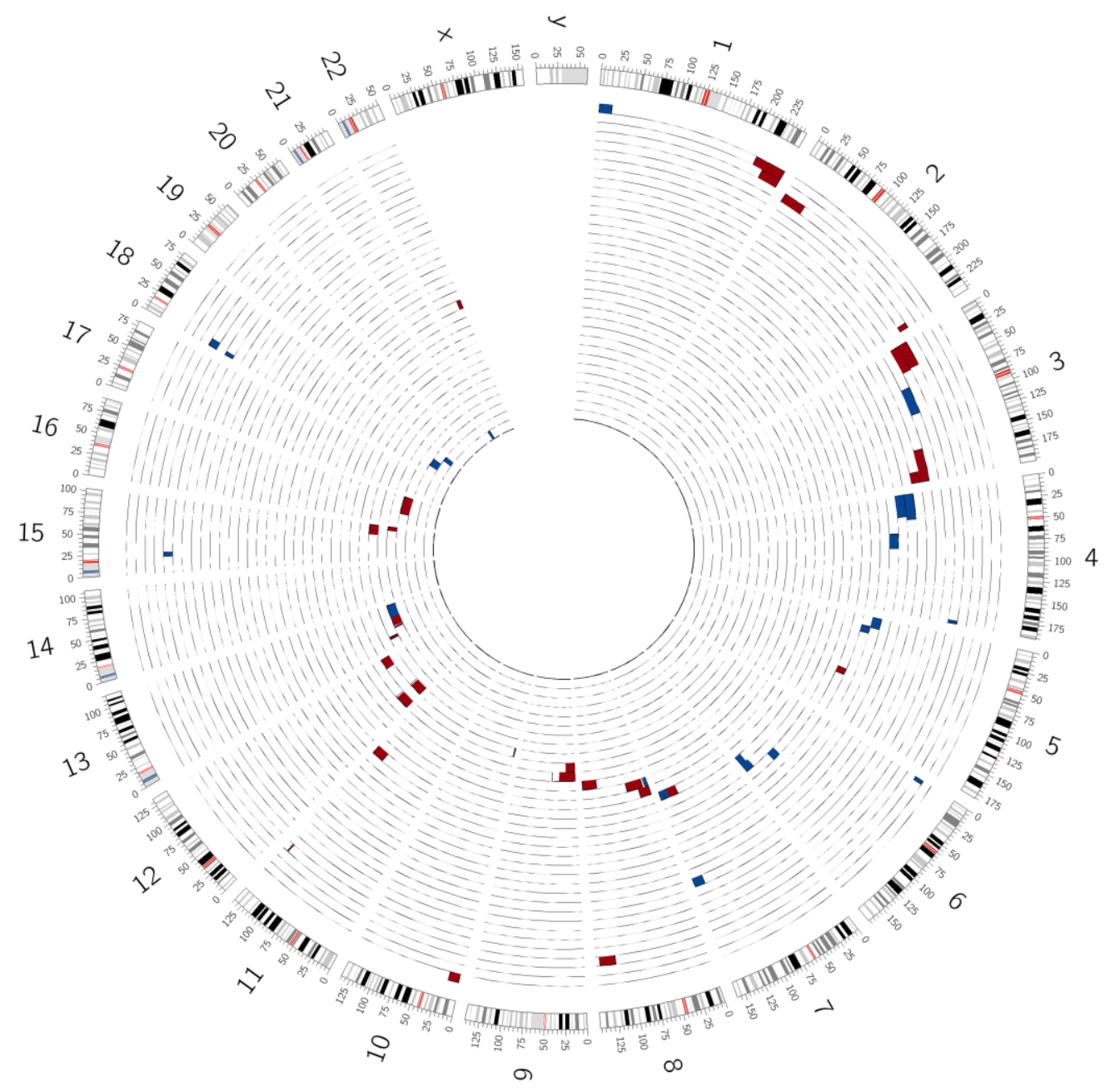

Figure 4. CNVs detected in the real clinical maternal plasma DNA samples carrying abnormal fetal DNA. Each sample is presented by an annular area between two adjacent circular lines. Amplifications and deletions are highlighted by red and blue, respectively. 


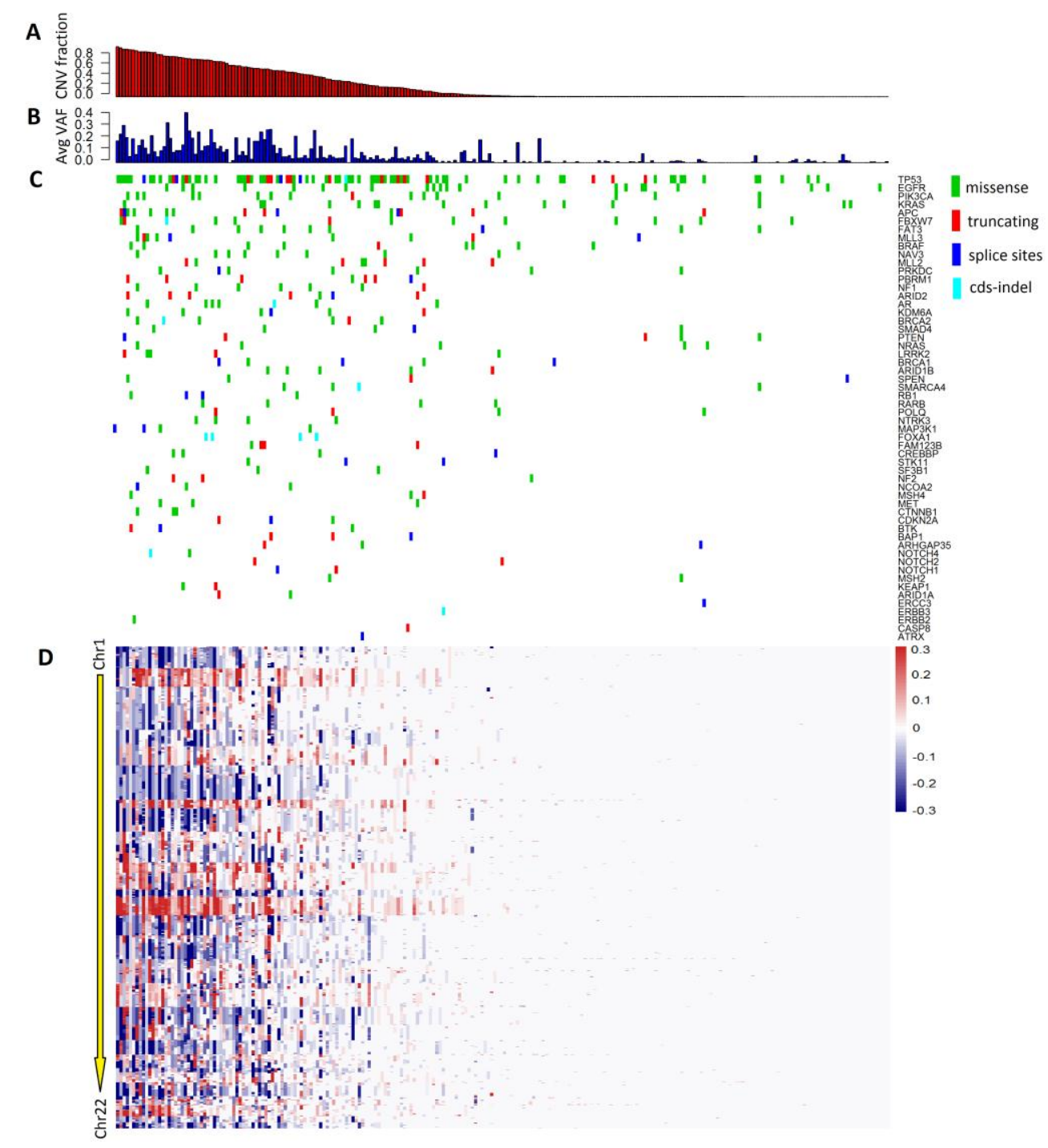

Figure 5. Mutations and CNVs detected in the cf-DNA of 240 cancer patients. (A) Fraction of CNV regions in the whole genome of each sample are showed by red bars. (B) Average variant allele frequencies (VAFs) of detected mutations in each sample are showed by blue bars. (C) Mutation spectrum of 240 cf-DNA samples. (D) CNV spectrum of 240 cf-DNA samples, ordered by genome positions from chromosome1 (top) to chromosome22 (bottom). Gains and losses were detected with the cutoff of $\mathrm{p}<10 \mathrm{e}-5$, and highlighted by red and blue, respectively. 

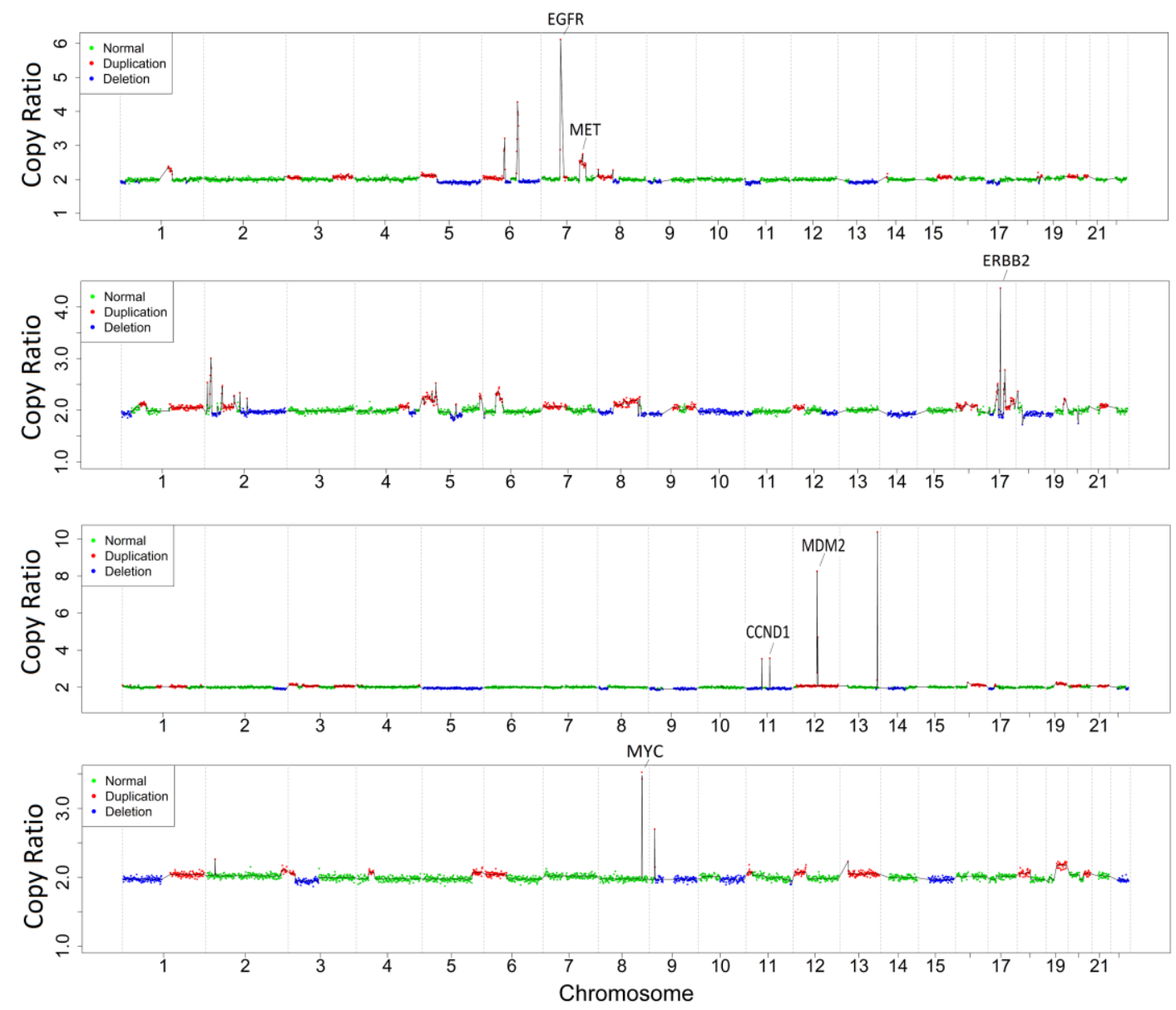

Figure 6. Examples of clinical relevant CNVs in cf-DNA of cancer patients. Gains and losses are highlighted by red and blue, respectively. The average VAF of mutations in four samples (from top to bottom) were $3.40 \%(n=11), 3.78 \%(n=24), 3.45 \%(n=5), 4.05 \%(n=4)$, respectively. 


\section{Supplementary Methods}

\section{Parameters for BIC-seq, DNAcopy, Control-FREEC and CNV-seq}

For BIC-seq, the initial bin size was set to $1 \mathrm{M}$ and the penalty parameter was chosen as 1.5. We chose the candidate CNVs as regions with P-values less than 0.01 and $\log \mathrm{R}>0.05$ or $<-0.05$. Default parameters were used for DNAcopy. For Control-FREEC, the parameter setting include window $=1000000$, degree $=4$, breakPointThreshold=2.0 and forceGCcontentNormalization $=1$. For CNV-seq, $0.75 \mathrm{M}$ was chosen as the windows size and the parameter of global-normalization was used. The other parameters include minimum-windows-required $=6$ and $\log 2$-threshold changing from 0.01 to 0.1 for obtaining different levels of sensitivity.

\section{Estimation of ct-DNA fraction in cf-DNA}

Considering that homozygous deletion of long DNA fragment (>10M) was not likely to occurred in cancer genomes, so we estimate the ct-DNA fraction using the lowest copy ratio of long DNA fragment deletions ( $>10 \mathrm{M})$ with $p<10 \mathrm{e}-5$. Assuming that $\mu$ is the lowest copy ratio of long DNA fragment deletions, we estimate the ct-DNA fraction to be $(1-\mu) \times 2$. If no long DNA fragment deletions is detected with $p<10 \mathrm{e}-5$, the ct-DNA fraction was estimated to be 0 .

\section{Test dataset}

The sequence data (fastq) have been deposited in the NCBI SRA database with the accession number of SRA525461. 


\section{Supplementary Figures:}
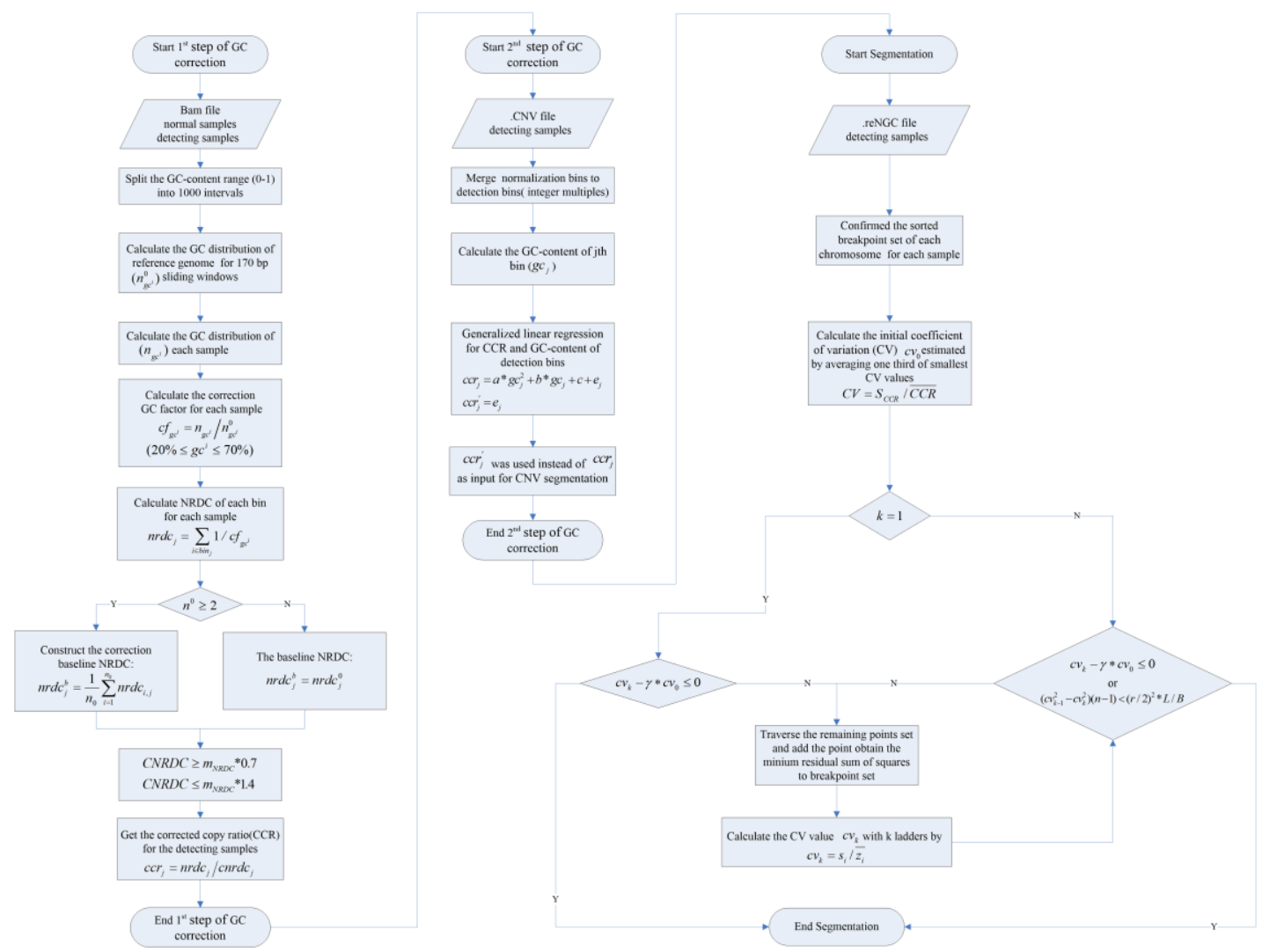

Figure S1. Workflow of SCDT. 

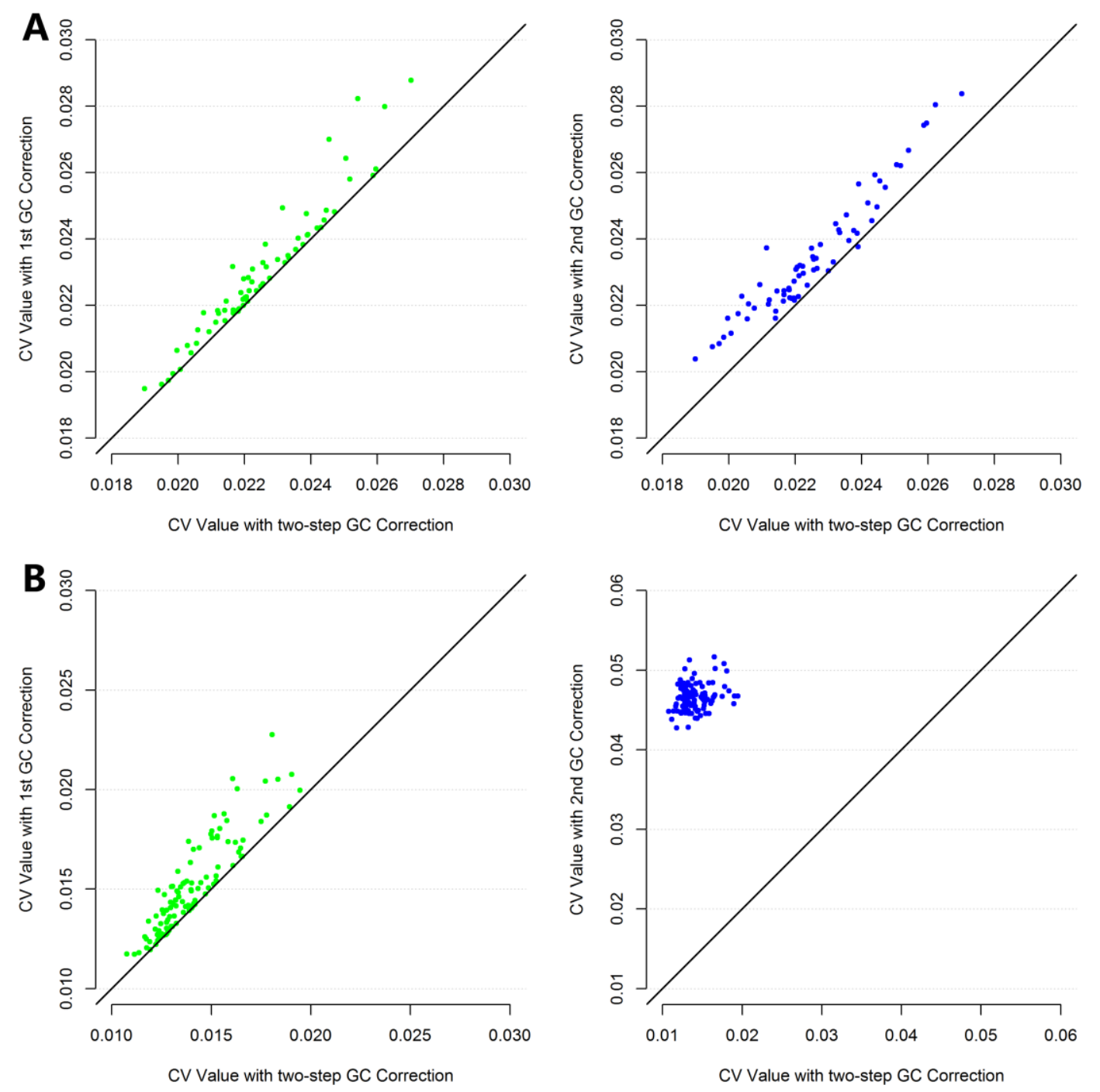

Figure S2. Comparison of CVs of copy ratios between two-step GC correction and one-step GC correction. CV of copy ratios after two-step GC correction (horizontal-axis) and only the first step GC correction (vertical-axis in the left panels) or only the second step GC correction (vertical-axis in the right panels) on the low-depth whole genome sequencing (WGS) data of 67 normal maternal plasma samples(A) or on the 138 target sequencing of normal samples(B). 


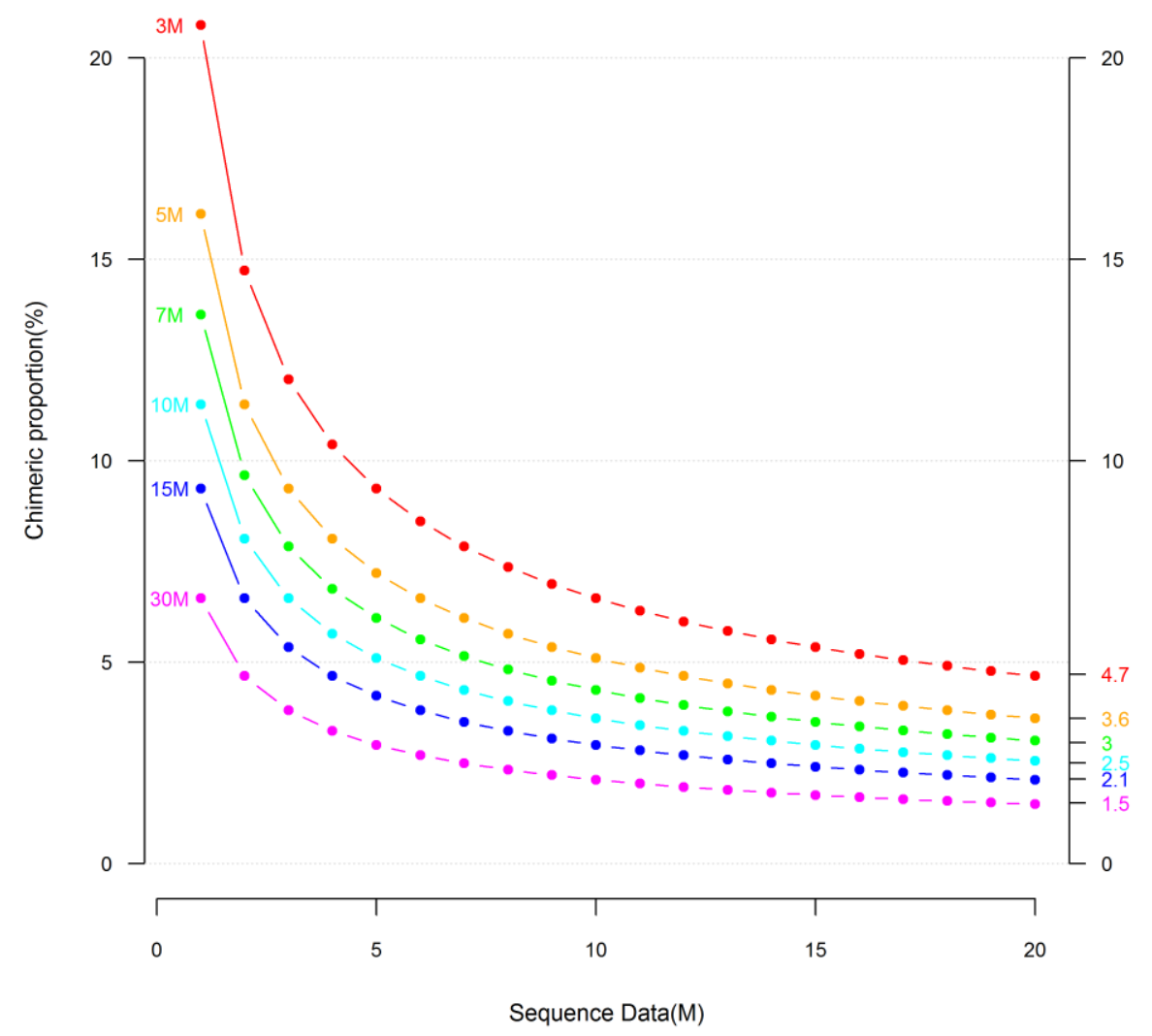

Figure S3. The theoretical CNV detection power determined by reads numbers, CNV length and the chimeric fraction of CNVs. The default confidence level is 0.001 and the size of test bin was set to $1 \mathrm{M}$. Lines of different colors indicate the minimal detectable chimeric proportion with certain reads number and $\mathrm{CNV}$ length. The right vertical axis indicates the minimal detectable chimeric proportion of target $\mathrm{CNV}$ with 20 million reads. 


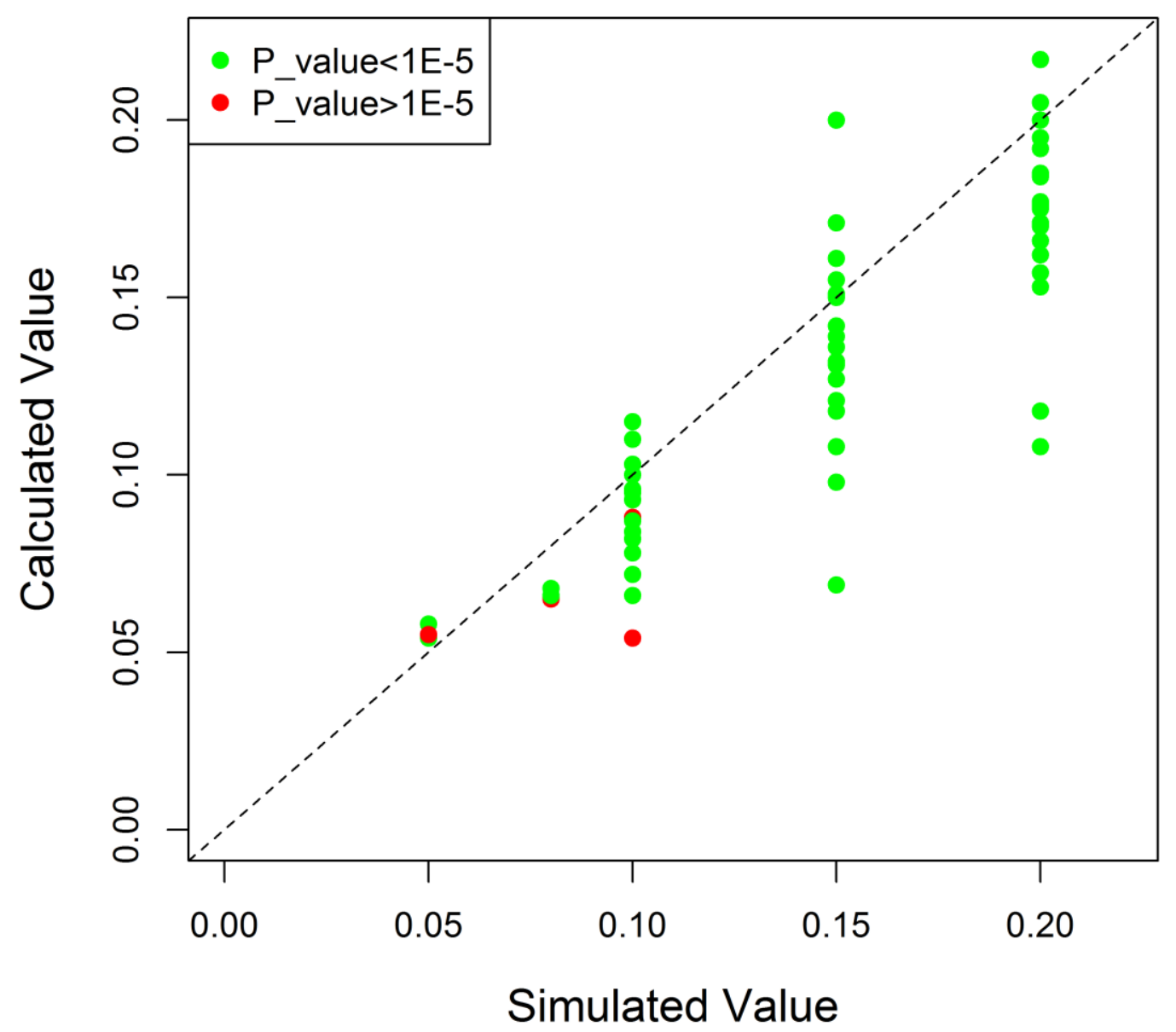

Figure S4. Evaluated chimeric fractions of the simulated spike-in CNVs. 

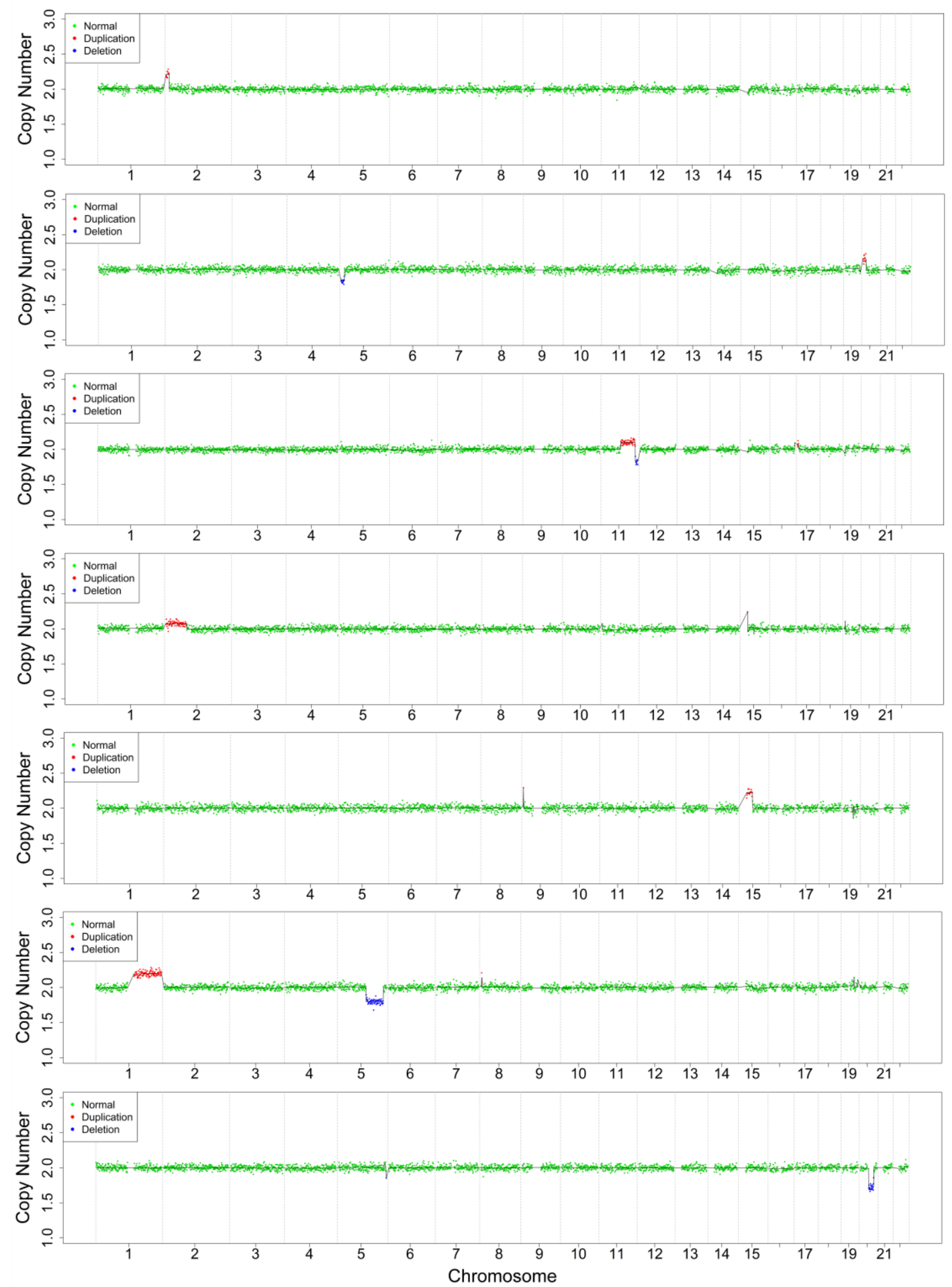

Figure S5. CNV profiles of 7 real maternal plasma samples which were identified to be normal by amniocentesis. Copy number gains and losses were highlighted by red and blue, respectively. 


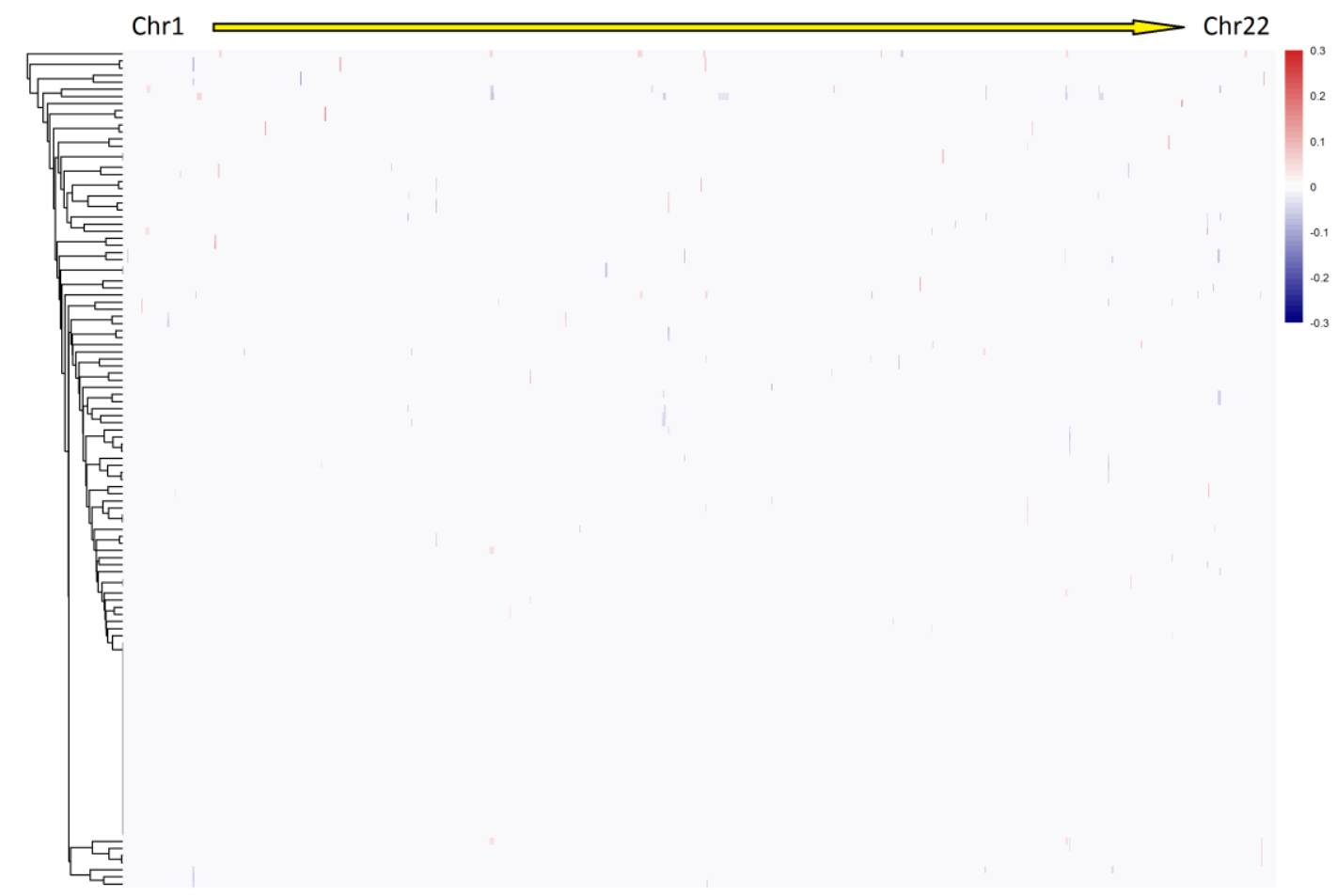

Figure S6. CNV spectrum in 138 target sequencing of normal cf-DNA samples, ordered by genome positions from chromosome1 (left) to chromosome22 (right). Gains and losses were detected with the cutoff of $\mathrm{p}<10 \mathrm{e}-5$, and highlighted by red and blue, respectively. 
bioRxiv preprint doi: https://doi.org/10.1101/411256; this version posted September 7, 2018. The copyright holder for this preprint (which was not certified by peer review) is the author/funder, who has granted bioRxiv a license to display the preprint in perpetuity. It is made available under aCC-BY-NC-ND 4.0 International license.

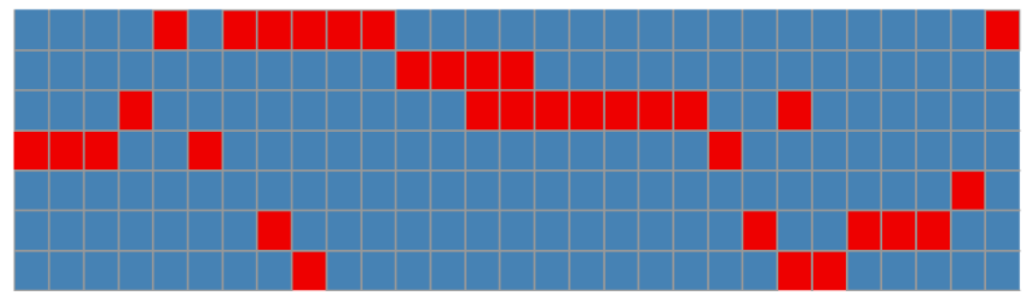

CCND1

CDK4

EGFR

ERBB2

FGFR1

KRAS

MET

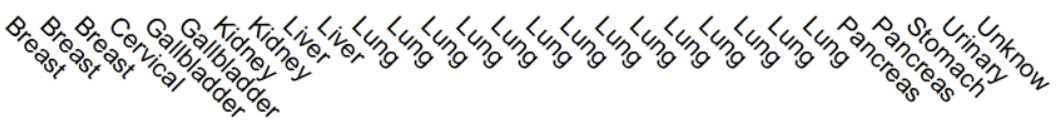

Figure S7. Targetable high level amplifications $(\mathrm{CN}>7)$ in cf-DNA of cancer patients. 
bioRxiv preprint doi: https://doi.org/10.1101/411256; this version posted September 7, 2018. The copyright holder for this preprint (which

was not certified by peer review) is the author/funder, who has granted bioRxiv a license to display the preprint in perpetuity. It is made available under aCC-BY-NC-ND 4.0 International license.

\section{Supplementary Tables}

\section{Supplementary table 1}

\begin{tabular}{|c|c|c|c|c|c|c|c|c|c|c|}
\hline Sample & Chr & Start & End & Length & Estimate_copy_ratio & P_value & Type & \begin{tabular}{|l|} 
Validated \\
\end{tabular} & Spike-in Ftaction & Theorical_P_value \\
\hline CL100005923_L01_17 & 1 & $2.00 \mathrm{E}+06$ & 8999999 & $7.00 \mathrm{E}+06$ & 0.898607539 & $7.88 \mathrm{E}-108$ & Del & YES & 0.2 & $1.63 \mathrm{E}-136$ \\
\hline CL100005923_L01_17 & 1 & $1.00 \mathrm{E}+06$ & $2.00 \mathrm{E}+06$ & $1.00 \mathrm{E}+06$ & 0.921543529 & $2.24 \mathrm{E}-10$ & Del & YES & 0.2 & $1.63 \mathrm{E}-136$ \\
\hline CL100005923_L01_18 & 1 & $1.00 \mathrm{E}+06$ & 8999999 & $8.00 \mathrm{E}+06$ & 0.922911807 & $3.34 \mathrm{E}-45$ & Del & YES & 0.15 & $1.56 \mathrm{E}-48$ \\
\hline CL100005923_L01_19 & 1 & $1.00 \mathrm{E}+06$ & 8999999 & $8.00 \mathrm{E}+06$ & 0.94540306 & $2.21 \mathrm{E}-20$ & Del & YES & 0.1 & $5.84 \mathrm{E}-19$ \\
\hline CL100005923_L02_1 & 8 & 0 & 6999999 & $7.00 \mathrm{E}+06$ & 1.096399256 & 0 & Dup & YES & 0.2 & 0 \\
\hline CL100005923_L02_1 & 4 & 0 & $9.00 \mathrm{E}+06$ & $9.00 \mathrm{E}+06$ & 0.901840503 & 1.75E-121 & Del & YES & 0.2 & $2.20 \mathrm{E}-142$ \\
\hline CL100005923_L02_-12 & 5 & 0 & 11999999 & $1.20 \mathrm{E}+07$ & 0.946974904 & $2.17 \mathrm{E}-27$ & Del & YES & 0.2 & $7.19 \mathrm{E}-113$ \\
\hline CL100005923_L02_13 & 5 & 0 & 11999999 & $1.20 \mathrm{E}+07$ & 0.965571992 & $4.51 \mathrm{E}-12$ & Del & YES & 0.15 & $6.31 \mathrm{E}-74$ \\
\hline CL100005923_L02_15 & 5 & 0 & 9999999 & $1.00 \mathrm{E}+07$ & 0.972481006 & $3.66 \mathrm{E}-07$ & Del & YES & 0.05 & $1.70 \mathrm{E}-06$ \\
\hline CL100005923_L02_17 & 22 & $1.90 \mathrm{E}+07$ & 20999999 & $2.00 \mathrm{E}+06$ & 0.938785193 & 2.02E-09 & Del & YES & 0.2 & $1.31 \mathrm{E}-30$ \\
\hline CL100005923_L02_2 & 8 & 0 & 6999999 & $7.00 \mathrm{E}+06$ & 1.071508954 & 0 & Dup & YES & 0.15 & 0 \\
\hline CL100005923_L02_2 & 4 & 0 & 8999999 & $9.00 \mathrm{E}+06$ & 0.924526802 & $1.56 \mathrm{E}-63$ & Del & YES & 0.15 & 2.22E-70 \\
\hline CL100005923_L02_3 & 8 & 0 & 6999999 & $7.00 \mathrm{E}+06$ & 1.04682088 & 0 & Dup & YES & 0.1 & 0 \\
\hline CL100005923_L02_3 & 4 & 0 & 10999999 & $1.10 \mathrm{E}+07$ & 0.955535745 & $1.10 \mathrm{E}-25$ & Del & YES & 0.1 & $2.15 \mathrm{E}-34$ \\
\hline CL100005923_L02_4 & 4 & $2.00 \mathrm{E}+06$ & 8999999 & $7.00 \mathrm{E}+06$ & 0.969164158 & 2.17E-08 & Del & YES & 0.05 & 1.03E-06 \\
\hline CL100005923_L02_4 & 8 & 0 & 4999999 & $5.00 \mathrm{E}+06$ & 1.027246911 & $6.37 \mathrm{E}-05$ & Dup & YES & 0.05 & 4.03E-05 \\
\hline CL100005923_L02_6 & 15 & $2.30 \mathrm{E}+07$ & 28999999 & $6.00 \mathrm{E}+06$ & 0.897981514 & $9.36 \mathrm{E}-87$ & Del & YES & 0.2 & 2.82E-70 \\
\hline CL100005923_L02_7 & 15 & $2.30 \mathrm{E}+07$ & 28999999 & $6.00 \mathrm{E}+06$ & 0.923807773 & $8.18 \mathrm{E}-66$ & Del & YES & 0.15 & $8.15 E-54$ \\
\hline CL100005923_L02_9 & 15 & $2.30 \mathrm{E}+07$ & 28999999 & $6.00 \mathrm{E}+06$ & 0.952274325 & $3.91 \mathrm{E}-19$ & Del & YES & 0.1 & 3.32E-18 \\
\hline CL100006330_L01_1 & 4 & 0 & 5999999 & $6.00 \mathrm{E}+06$ & 0.914280779 & $5.95 E-75$ & Del & YES & 0.2 & $2.35 \mathrm{E}-100$ \\
\hline CL100006330_L01_12 & 7 & $7.30 \mathrm{E}+07$ & 73999999 & $1.00 \mathrm{E}+06$ & 0.937111848 & 4.23E-06 & Del & YES & 0.15 & $2.57 \mathrm{E}-12$ \\
\hline CL100006330_L01_17 & 5 & 0 & 999999 & $1.00 \mathrm{E}+06$ & 0.945340937 & 0.0001113 & Del & YES & 0.05 & $9.44 \mathrm{E}-06$ \\
\hline CL100006330_L01_2 & 4 & 0 & 4999999 & $5.00 \mathrm{E}+06$ & 0.94288694 & $3.97 \mathrm{E}-22$ & Del & YES & 0.15 & $6.13 \mathrm{E}-46$ \\
\hline CL100006330_L01_20 & 4 & 0 & 5999999 & $6.00 \mathrm{E}+06$ & 0.922463126 & $2.15 E-48$ & Del & YES & 0.2 & $1.36 \mathrm{E}-80$ \\
\hline CL100006330_L01_3 & 4 & 0 & 5999999 & $6.00 \mathrm{E}+06$ & 0.956993805 & $1.67 \mathrm{E}-12$ & Del & YES & 0.1 & 8.17E-17 \\
\hline CL100006330_L01_6 & 5 & $1.00 \mathrm{E}+06$ & 23999999 & $2.30 \mathrm{E}+07$ & 0.912873622 & $2.10 \mathrm{E}-197$ & Del & YES & 0.2 & 2.35E-279 \\
\hline CL100006330_L01_6 & 5 & 0 & $1.00 \mathrm{E}+06$ & $1.00 \mathrm{E}+06$ & 0.877299161 & $3.56 \mathrm{E}-20$ & Del & YES & 0.2 & 2.35E-279 \\
\hline CL100006330_L01_7 & 5 & 0 & 23999999 & $2.40 \mathrm{E}+07$ & 0.932907537 & $4.29 \mathrm{E}-125$ & Del & YES & 0.15 & $2.14 \mathrm{E}-161$ \\
\hline CL100006330_L01_8 & 5 & $5.00 \mathrm{E}+06$ & 23999999 & $1.90 \mathrm{E}+07$ & 0.957997386 & $3.27 \mathrm{E}-31$ & Del & YES & 0.1 & 1.40E-59 \\
\hline CL100006330_L02_11 & 15 & $2.40 \mathrm{E}+07$ & 28999999 & $5.00 \mathrm{E}+06$ & 0.905775183 & $2.05 \mathrm{E}-60$ & Del & YES & 0.2 & $3.08 \mathrm{E}-86$ \\
\hline CL100006330_L02_11 & 15 & $2.30 \mathrm{E}+07$ & $2.40 \mathrm{E}+07$ & $1.00 \mathrm{E}+06$ & 0.94011912 & 7.41E-06 & Del & YES & 0.2 & $3.08 \mathrm{E}-86$ \\
\hline CL100006330_L02_12 & 15 & $2.30 \mathrm{E}+07$ & 28999999 & $6.00 \mathrm{E}+06$ & 0.936739358 & $8.15 E-29$ & Del & YES & 0.15 & 4.57E-44 \\
\hline CL100006330_L02_13 & 15 & $2.30 \mathrm{E}+07$ & 28999999 & $6.00 \mathrm{E}+06$ & 0.965064307 & 8.34E-07 & Del & YES & 0.1 & $1.56 \mathrm{E}-15$ \\
\hline CL100006330_L02_16 & 22 & $1.90 \mathrm{E}+07$ & $2.10 \mathrm{E}+07$ & $2.00 \mathrm{E}+06$ & 0.910399169 & $6.53 \mathrm{E}-27$ & Del & YES & 0.2 & $1.46 \mathrm{E}-43$ \\
\hline CL100006330_L02_16 & 22 & $2.10 \mathrm{E}+07$ & 21999999 & $1.00 \mathrm{E}+06$ & 0.935011032 & $9.17 \mathrm{E}-08$ & Del & YES & 0.2 & $1.46 \mathrm{E}-43$ \\
\hline CL100006330_L02_17 & 22 & $1.90 \mathrm{E}+07$ & 21999999 & $3.00 \mathrm{E}+06$ & 0.941845293 & $7.20 \mathrm{E}-14$ & Del & YES & 0.15 & $6.55 \mathrm{E}-21$ \\
\hline CL100006330_L02_18 & 22 & $1.70 \mathrm{E}+07$ & 20999999 & $4.00 \mathrm{E}+06$ & 0.964708536 & $9.38 \mathrm{E}-06$ & Del & YES & 0.1 & $2.29 \mathrm{E}-08$ \\
\hline CL100006330_L02_6 & 15 & $2.40 \mathrm{E}+07$ & 28999999 & $5.00 \mathrm{E}+06$ & 0.901724201 & $2.40 \mathrm{E}-75$ & Del & YES & 0.2 & $8.53 E-83$ \\
\hline CL100006330_L02_6 & 15 & $2.30 \mathrm{E}+07$ & $2.40 \mathrm{E}+07$ & $1.00 \mathrm{E}+06$ & 0.939121122 & $1.15 \mathrm{E}-06$ & Del & YES & 0.2 & 8.53E-83 \\
\hline CL100006330_L02_7 & 15 & $2.30 \mathrm{E}+07$ & 28999999 & $6.00 \mathrm{E}+06$ & 0.929928128 & $8.52 \mathrm{E}-46$ & Del & YES & 0.15 & $2.72 \mathrm{E}-47$ \\
\hline CL100006330_L02_8 & 15 & $2.30 \mathrm{E}+07$ & 27999999 & $5.00 \mathrm{E}+06$ & 0.963597874 & 1.44E-08 & Del & YES & 0.1 & $2.48 \mathrm{E}-18$ \\
\hline CL100014014_L01_11 & 17 & $1.70 \mathrm{E}+07$ & $1.80 \mathrm{E}+07$ & $1.00 \mathrm{E}+06$ & 0.880599301 & $2.50 \mathrm{E}-24$ & Del & YES & 0.2 & $2.64 \mathrm{E}-57$ \\
\hline CL100014014_L01_11 & 17 & $1.90 \mathrm{E}+07$ & 19999999 & $1.00 \mathrm{E}+06$ & 0.919495266 & $3.62 \mathrm{E}-11$ & Del & YES & 0.2 & $2.64 \mathrm{E}-57$ \\
\hline CL100014014_L01_13 & 17 & $1.70 \mathrm{E}+07$ & 19999999 & $3.00 \mathrm{E}+06$ & 0.911354222 & $2.48 \mathrm{E}-21$ & Del & YES & 0.15 & $3.45 \mathrm{E}-26$ \\
\hline CL100014014_L01_16 & 15 & $2.50 \mathrm{E}+07$ & 28999999 & $4.00 \mathrm{E}+06$ & 0.917197011 & $7.35 \mathrm{E}-46$ & Del & YES & 0.2 & 7.46E-97 \\
\hline CL100014014_L01_16 & 15 & $2.30 \mathrm{E}+07$ & $2.50 \mathrm{E}+07$ & $2.00 \mathrm{E}+06$ & 0.932232947 & $9.43 \mathrm{E}-16$ & Del & YES & 0.2 & $7.46 \mathrm{E}-97$ \\
\hline CL100014014_L01_17 & 15 & $2.30 \mathrm{E}+07$ & $2.90 \mathrm{E}+07$ & $6.00 \mathrm{E}+06$ & 0.950646161 & $9.11 \mathrm{E}-19$ & Del & YES & 0.15 & $2.60 \mathrm{E}-45$ \\
\hline CL100014014_L01_6 & 15 & $2.40 \mathrm{E}+07$ & 28999999 & $5.00 \mathrm{E}+06$ & 0.909972719 & $4.52 \mathrm{E}-56$ & Del & YES & 0.2 & 3.24E-73 \\
\hline CL100014014_L01_6 & 15 & $2.30 \mathrm{E}+07$ & $2.40 \mathrm{E}+07$ & $1.00 \mathrm{E}+06$ & 0.941341581 & 9.43E-06 & Del & YES & 0.2 & 3.24E-73 \\
\hline CL100014014_L01_7 & 15 & $2.40 \mathrm{E}+07$ & 28999999 & $5.00 \mathrm{E}+06$ & 0.945451827 & 3.43E-16 & Del & YES & 0.15 & $1.32 \mathrm{E}-33$ \\
\hline CL100014014_L01_8 & 15 & $2.30 \mathrm{E}+07$ & $3.00 \mathrm{E}+07$ & $7.00 \mathrm{E}+06$ & 0.972777748 & $2.85 \mathrm{E}-05$ & Del & YES & 0.08 & $3.68 \mathrm{E}-10$ \\
\hline CL100016513_L01_1 & 9 & $1.29 \mathrm{E}+08$ & $1.40 \mathrm{E}+08$ & 1.10E+07 & 1.08079154 & 0 & Dup & YES & 0.2 & 0 \\
\hline CL100016513_L01_1 & 9 & $1.40 \mathrm{E}+08$ & 140999999 & $1.00 \mathrm{E}+06$ & 1.101131118 & 0 & Dup & YES & 0.2 & 0 \\
\hline CL100016513_L01_1 & 1 & $2.00 E+06$ & 3999999 & $2.00 \mathrm{E}+06$ & 0.891927582 & $1.31 \mathrm{E}-41$ & Del & YES & 0.2 & $2.59 \mathrm{E}-69$ \\
\hline CL100016513_L01_1 & 1 & $1.00 \mathrm{E}+06$ & $2.00 \mathrm{E}+06$ & $1.00 \mathrm{E}+06$ & 0.920281493 & $6.58 \mathrm{E}-12$ & Del & YES & 0.2 & $2.59 \mathrm{E}-69$ \\
\hline CL100016513_L01_10 & 15 & $2.30 \mathrm{E}+07$ & 28999999 & $6.00 \mathrm{E}+06$ & 0.897245554 & 1.76E-30 & Del & YES & 0.15 & 2.93E-16 \\
\hline CL100016513_L01_11 & 15 & $2.50 \mathrm{E}+07$ & 27999999 & $3.00 \mathrm{E}+06$ & 0.945095742 & $1.08 \mathrm{E}-12$ & Del & YES & 0.1 & $1.76 \mathrm{E}-19$ \\
\hline CL100016513_L01_11 & 15 & $2.30 \mathrm{E}+07$ & $2.40 \mathrm{E}+07$ & $1.00 \mathrm{E}+06$ & 0.917003889 & $2.17 \mathrm{E}-11$ & Del & YES & 0.1 & $1.76 \mathrm{E}-19$ \\
\hline CL100016513_L01_12 & 15 & $2.30 \mathrm{E}+07$ & 27999999 & $5.00 \mathrm{E}+06$ & 0.967740085 & $6.13 \mathrm{E}-05$ & Del & YES & 0.08 & $4.85 \mathrm{E}-09$ \\
\hline CL100016513_L01_17 & 15 & $2.40 \mathrm{E}+07$ & 28999999 & $5.00 \mathrm{E}+06$ & 0.902541467 & 4.46E-71 & Del & YES & 0.2 & $3.01 \mathrm{E}-80$ \\
\hline CL100016513_L01_18 & 15 & $2.40 \mathrm{E}+07$ & 28999999 & $5.00 \mathrm{E}+06$ & 0.933047035 & $9.02 \mathrm{E}-32$ & Del & YES & 0.15 & $1.73 \mathrm{E}-43$ \\
\hline CL100016513_L01_19 & 15 & $2.30 \mathrm{E}+07$ & 28999999 & $6.00 \mathrm{E}+06$ & 0.956741294 & $2.61 \mathrm{E}-12$ & Del & YES & 0.1 & $2.33 \mathrm{E}-15$ \\
\hline CL100016513_L01_2 & 9 & $1.29 \mathrm{E}+08$ & 140999999 & $1.20 \mathrm{E}+07$ & 1.069787048 & 0 & Dup & YES & 0.15 & 0 \\
\hline CL100016513_L01_2 & 1 & $2.00 \mathrm{E}+06$ & 3999999 & $2.00 \mathrm{E}+06$ & 0.909694076 & $1.47 \mathrm{E}-26$ & Del & YES & 0.15 & $1.65 \mathrm{E}-34$ \\
\hline CL100016513_L01_2 & 1 & $1.00 \mathrm{E}+06$ & $2.00 \mathrm{E}+06$ & $1.00 \mathrm{E}+06$ & 0.941487342 & 2.97E-06 & Del & YES & 0.15 & $1.65 \mathrm{E}-34$ \\
\hline CL100016513_L01_20 & 15 & $2.30 \mathrm{E}+07$ & 28999999 & $6.00 \mathrm{E}+06$ & 0.965499419 & 2.99E-10 & Del & YES & 0.08 & 4.09E-13 \\
\hline CL100016513_L01_3 & 9 & $1.33 E+08$ & 140999999 & $8.00 \mathrm{E}+06$ & 1.054394196 & 0 & Dup & YES & 0.1 & 0 \\
\hline CL100016513_L01_3 & 1 & $1.00 \mathrm{E}+06$ & 3999999 & $3.00 \mathrm{E}+06$ & 0.946604677 & $2.78 \mathrm{E}-14$ & Del & YES & 0.1 & $4.43 \mathrm{E}-16$ \\
\hline CL100016513_L01_3 & 9 & $1.29 \mathrm{E}+08$ & $1.33 \mathrm{E}+08$ & $4.00 \mathrm{E}+06$ & 1.03967374 & $1.46 \mathrm{E}-09$ & Dup & YES & 0.1 & 0 \\
\hline CL100016513_L01_4 & 9 & $1.36 \mathrm{E}+08$ & 140999999 & $5.00 \mathrm{E}+06$ & 1.039936108 & 7.79E-13 & Dup & YES & 0.08 & 0 \\
\hline CL100016513_L01_4 & 9 & $1.29 \mathrm{E}+08$ & $1.36 \mathrm{E}+08$ & $7.00 \mathrm{E}+06$ & 1.028794894 & $7.55 \mathrm{E}-08$ & Dup & YES & 0.08 & 0 \\
\hline CL100016513_L01_8 & 15 & $2.30 \mathrm{E}+07$ & 28999999 & $6.00 \mathrm{E}+06$ & 0.912956061 & 1.15E-45 & Del & YES & 0.2 & $6.09 \mathrm{E}-63$ \\
\hline
\end{tabular}


bioRxiv preprint doi: https://doi.org/10.1101/411256; this version posted September 7, 2018. The copyright holder for this preprint (which was not certified by peer review) is the author/funder, who has granted bioRxiv a license to display the preprint in perpetuity. It is made available under aCC-BY-NC-ND 4.0 International license.

Supplementary table 2

\begin{tabular}{|c|c|c|c|c|c|c|c|c|}
\hline Sample & Chr & Start & End & Length & Estimate_copy_ratio & P_value & Type & Validated \\
\hline CL100016513_L02_31 & 5 & 0 & 17999999 & $1.80 \mathrm{E}+07$ & 0.887501211 & $1.39 \mathrm{E}-137$ & Del & Yes \\
\hline CL100016513_L02_33 & 9 & 0 & 38999999 & $3.90 \mathrm{E}+07$ & 1.104866778 & 0 & Dup & Yes \\
\hline CL100016513_L02_37 & 18 & $6.10 \mathrm{E}+07$ & 77999999 & $1.70 \mathrm{E}+07$ & 0.900977684 & 3.62E-106 & Del & Yes \\
\hline CL100016513_L02_38 & 3 & 0 & 34999999 & $3.50 \mathrm{E}+07$ & 1.05307488 & 0 & Dup & Yes \\
\hline CL100016513_L02_42 & 6 & $1.03 \mathrm{E}+08$ & 117999999 & $1.50 \mathrm{E}+07$ & 0.938366647 & $2.52 E-34$ & Del & Yes \\
\hline CL100017066_L01_12 & 3 & $1.82 \mathrm{E}+08$ & 197999999 & $1.60 \mathrm{E}+07$ & 1.134271942 & 0 & Dup & Yes \\
\hline CL100017066_L01_12 & 7 & $1.43 \mathrm{E}+08$ & 158999999 & $1.60 \mathrm{E}+07$ & 0.867450059 & $6.58 \mathrm{E}-177$ & Del & Yes \\
\hline CL100017066_L01_13 & 3 & 0 & 36999999 & $3.70 \mathrm{E}+07$ & 1.042350453 & 0 & Dup & Yes \\
\hline CL100017066_L01_13 & 18 & 0 & 4999999 & $5.00 \mathrm{E}+06$ & 0.947326212 & $2.38 \mathrm{E}-08$ & Del & Yes \\
\hline CL100017066_L01_14 & 11 & $1.10 \mathrm{E}+08$ & 134999999 & $2.50 \mathrm{E}+07$ & 1.04564861 & 0 & Dup & Yes \\
\hline CL100017066_L01_14 & 6 & $1.59 \mathrm{E}+08$ & 170999999 & $1.20 \mathrm{E}+07$ & 0.964109986 & 4.04E-09 & Del & Yes \\
\hline CL100017066_L01_15 & 6 & $1.62 \mathrm{E}+08$ & 170999999 & $9.00 \mathrm{E}+06$ & 0.862799059 & $2.12 \mathrm{E}-146$ & Del & Yes \\
\hline CL100017066_L01_15 & 6 & $1.54 \mathrm{E}+08$ & $1.62 \mathrm{E}+08$ & $8.00 \mathrm{E}+06$ & 0.940716773 & 2.67E-21 & Del & Yes \\
\hline CL100017066_L01_18 & 5 & $1.02 E+08$ & 112999999 & $1.10 \mathrm{E}+07$ & 1.054948746 & 0 & Dup & Yes \\
\hline CL100017066_L01_20 & 2 & $2.36 \mathrm{E}+08$ & 242999999 & $7.00 \mathrm{E}+06$ & 1.08772313 & 0 & Dup & Yes \\
\hline CL100017066_L01_20 & 18 & 0 & 9999999 & $1.00 \mathrm{E}+07$ & 0.905114039 & $1.76 \mathrm{E}-60$ & Del & Yes \\
\hline CL100017066_L01_3 & 12 & $1.07 \mathrm{E}+08$ & 133999999 & $2.70 \mathrm{E}+07$ & 1.06204632 & 0 & Dup & Yes \\
\hline CL100017066_L01_3 & 10 & 0 & 2999999 & $3.00 \mathrm{E}+06$ & 0.936940142 & $3.25 \mathrm{E}-13$ & Del & Yes \\
\hline CL100017066_L01_4 & 9 & 0 & 21999999 & $2.20 \mathrm{E}+07$ & 1.042862869 & 0 & Dup & Yes \\
\hline CL100017066_L01_4 & 12 & 0 & 33999999 & $3.40 \mathrm{E}+07$ & 1.046307159 & 0 & Dup & Yes \\
\hline CL100017066_L01_44 & 21 & $1.50 \mathrm{E}+07$ & 23999999 & $9.00 \mathrm{E}+06$ & 0.93609728 & $3.74 \mathrm{E}-25$ & Del & Yes \\
\hline CL100017066_L01_6 & 4 & $6.20 \mathrm{E}+07$ & 85999999 & $2.40 \mathrm{E}+07$ & 0.962856732 & $2.04 \mathrm{E}-17$ & Del & Yes \\
\hline CL100017066_L01_7 & 3 & $5.90 \mathrm{E}+07$ & 97999999 & $3.90 \mathrm{E}+07$ & 0.975397351 & $4.14 \mathrm{E}-08$ & Del & Yes \\
\hline CL100017066_L01_8 & 1 & $2.23 \mathrm{E}+08$ & 248999999 & $2.60 \mathrm{E}+07$ & 1.125025541 & 0 & Dup & Yes \\
\hline CL100017066_L01_8 & 6 & 0 & 4999999 & $5.00 \mathrm{E}+06$ & 0.883814707 & $1.66 \mathrm{E}-46$ & Del & Yes \\
\hline CL100017066_L01_9 & 3 & $1.50 \mathrm{E}+08$ & 197999999 & $4.80 \mathrm{E}+07$ & 5309 & 0 & Dup & Yes \\
\hline CL100017066_L02_21 & 13 & $2.00 \mathrm{E}+07$ & $2.40 \mathrm{E}+07$ & $4.00 \mathrm{E}+06$ & 1.070766305 & 0 & Dup & Yes \\
\hline CL100017066_L02_21 & 15 & $7.50 \mathrm{E}+07$ & 102999999 & $2.80 \mathrm{E}+07$ & 1.067374502 & 0 & Dup & Yes \\
\hline CL100017066_L02_21 & 13 & $2.50 \mathrm{E}+07$ & 26999999 & $2.00 \mathrm{E}+06$ & 1.052683185 & $3.72 \mathrm{E}-06$ & Dup & Yes \\
\hline CL100017066_L02_22 & 15 & $9.10 \mathrm{E}+07$ & 102999999 & $1.20 \mathrm{E}+07$ & 1.090749056 & 0 & Dup & Yes \\
\hline CL100017066_L02_23 & 13 & $5.30 \mathrm{E}+07$ & $7.50 \mathrm{E}+07$ & $2.20 \mathrm{E}+07$ & 1.049337076 & 0 & Dup & Yes \\
\hline CL100017066_L02_23 & 13 & $7.50 \mathrm{E}+07$ & $7.80 \mathrm{E}+07$ & $3.00 \mathrm{E}+06$ & 1.101681725 & 0 & Dup & Yes \\
\hline CL100017066_L02_23 & 13 & $7.80 \mathrm{E}+07$ & 114999999 & $3.70 \mathrm{E}+07$ & 5872676 & $2.49 \mathrm{E}-100$ & Del & Yes \\
\hline CL100017066_L02_23 & 13 & $4.50 \mathrm{E}+07$ & $4.90 \mathrm{E}+07$ & $4.00 \mathrm{E}+06$ & 1.054928849 & 3.43E-09 & Dup & Yes \\
\hline CL100017066_L02_24 & 5 & $1.70 \mathrm{E}+07$ & 28999999 & $1.20 \mathrm{E}+07$ & 0.918787306 & $2.39 \mathrm{E}-34$ & Del & Yes \\
\hline CL100017066_L02_25 & 8 & 0 & 22999999 & $2.30 \mathrm{E}+07$ & 1.063801078 & 0 & Dup & Yes \\
\hline CL100017066_L02_26 & 4 & 0 & 39999999 & $4.00 \mathrm{E}+07$ & 0.954084728 & $8.67 \mathrm{E}-43$ & Del & Yes \\
\hline CL100017066_L02_27 & 8 & $1.20 \mathrm{E}+07$ & 47999999 & $3.60 \mathrm{E}+07$ & 1.053063259 & 0 & Dup & Yes \\
\hline CL100017066_L02_27 & 8 & 0 & $7.00 \mathrm{E}+06$ & $E+06$ & 434 & 4.30E-19 & Del & Yes \\
\hline CL100017066_L02_29 & 10 & $2.00 \mathrm{E}+06$ & $1.50 \mathrm{E}+07$ & $1.30 \mathrm{E}+07$ & 1.072197238 & 0 & Dup & Yes \\
\hline CL100017066_L02_29 & 1 & $1.00 \mathrm{E}+06$ & 16999999 & $1.60 \mathrm{E}+07$ & 0.920613844 & 3.95E-81 & Del & Yes \\
\hline CL100017066_L02_32 & 7 & $1.22 \mathrm{E}+08$ & $1.42 \mathrm{E}+08$ & $2.00 \mathrm{E}+07$ & 1.055642174 & 0 & Dup & Yes \\
\hline CL100017066_L02_32 & 7 & $1.42 \mathrm{E}+08$ & $1.48 \mathrm{E}+08$ & $6.00 \mathrm{E}+06$ & 0.933183964 & 7.76E-25 & Del & Yes \\
\hline CL100017066_L02_32 & 7 & $1.52 \mathrm{E}+08$ & 158999999 & $7.00 \mathrm{E}+06$ & 0.948429319 & $3.25 E-16$ & Del & Yes \\
\hline CL100017066_L02_32 & 22 & $4.30 \mathrm{E}+07$ & 50999999 & $8.00 \mathrm{E}+06$ & 1.041045151 & $5.57 \mathrm{E}-11$ & Dup & No \\
\hline CL100017066_L02_32 & 7 & $1.50 \mathrm{E}+08$ & $1.52 \mathrm{E}+08$ & $2.00 \mathrm{E}+06$ & & $7.07 \mathrm{E}-11$ & Del & Yes \\
\hline CL100017066_L02_33 & 1 & $2.09 E+08$ & 248999999 & $4.00 \mathrm{E}+07$ & 1.058877287 & 0 & Dup & Yes \\
\hline CL100017066_L02_34 & 8 & $1.13 \mathrm{E}+08$ & 145999999 & $3.30 \mathrm{E}+07$ & 1.039174177 & 0 & Dup & Yes \\
\hline CL100017066_L02_35 & 4 & 0 & 34999999 & $3.50 \mathrm{E}+07$ & 0.946056263 & $1.77 \mathrm{E}-55$ & Del & Yes \\
\hline CL100017066_L02_36 & 18 & $2.00 \mathrm{E}+07$ & 45999999 & $2.60 \mathrm{E}+07$ & 0.954044289 & $1.72 \mathrm{E}-28$ & Del & Yes \\
\hline CL100017066_L02_37 & 2 & 0 & 30999999 & $3.10 \mathrm{E}+07$ & 1.068014316 & 0 & Dup & Yes \\
\hline CL100017066_L02_37 & 8 & $1.20 \mathrm{E}+08$ & $1.41 \mathrm{E}+08$ & $2.10 \mathrm{E}+07$ & 1.050465712 & 0 & Dup & Yes \\
\hline CL100017066_L02_38 & 16 & $3.40 \mathrm{E}+07$ & $7.80 \mathrm{E}+07$ & $4.40 \mathrm{E}+07$ & 1.071450552 & 0 & Dup & Yes \\
\hline CL100017066 L02 38 & 16 & $7.80 \mathrm{E}+07$ & 89999999 & $1.20 \mathrm{E}+07$ & 1.098995091 & 0 & Dup & Yes \\
\hline CL100017066_L02_45 & 12 & 0 & 33999999 & $3.40 \mathrm{E}+07$ & 1.054389375 & 0 & Dup & Yes \\
\hline
\end{tabular}


Supplementary table 3

\begin{tabular}{|c|c|c|c|c|}
\hline Sample & Cancer Type & $\begin{array}{l}\text { Number of } \\
\text { mutations }\end{array}$ & $\begin{array}{c}\text { Tumor fraction estimated } \\
\text { by } \mathrm{SNV} / \text { indel }\end{array}$ & $\begin{array}{l}\text { Tumor fraction } \\
\text { etimated by CNV }\end{array}$ \\
\hline 14P1008444 & Cardia & 7 & 0.209285714 & 0.351433966 \\
\hline 15P6653209-1 & Gallbladder & 0 & 0 & 0 \\
\hline 15P6653177-1 & Gallbladder & 9 & 0.203888889 & 0.24462597 \\
\hline 14P1011925-1 & Gallbladder & 17 & 0.051294118 & 0.089929658 \\
\hline 14P1113003 & Unknow & 4 & 0.04025 & 0.038773001 \\
\hline 15P6219946-1 & Lung & 0 & 0 & 0 \\
\hline 15P6226246-1 & Lung & 1 & 0.012 & 0 \\
\hline 15P6653214-1 & Lung & 0 & 0 & 0 \\
\hline 15P6653255-1 & Lung & 1 & 0.012 & 0 \\
\hline 15P6658474-1 & Lung & 2 & 0.0255 & 0 \\
\hline 14P1006997 & Lung & 0 & 0 & 0 \\
\hline 14P1114097-1 & Lung & 0 & 0 & 0 \\
\hline 14P1114529-1 & Lung & 0 & 0 & 0 \\
\hline 15P0201526-1 & Lung & 0 & 0 & 0 \\
\hline 15P0201532-1 & Lung & 0 & 0 & 0 \\
\hline 15P6219948-1 & Lung & 0 & 0 & 0 \\
\hline 15P6226220-1 & Lung & 0 & 0 & 0 \\
\hline 15P6651893-1 & Lung & 1 & 0.014 & 0 \\
\hline 15P6652494-1 & Lung & 0 & 0 & 0 \\
\hline 15P6653243-1 & Lung & 0 & 0 & 0 \\
\hline 15P6615698-1 & Lung & 0 & 0 & 0 \\
\hline 15P6651217-1 & Lung & 6 & 0.061666667 & 0.080435927 \\
\hline 15P6653199-1 & Lung & 0 & 0 & 0 \\
\hline 15P6653269-1 & Lung & 7 & 0.040285714 & 0.052360092 \\
\hline 14P1113568 & Lung & 0 & 0 & 0.032423453 \\
\hline 15P6658590-1 & Lung & 0 & 0 & 0.025570068 \\
\hline 15P6658735-1 & Lung & 4 & 0.05975 & 0.071655274 \\
\hline 15P6652529-1 & Lung & 3 & 0.035666667 & 0.065412934 \\
\hline 14P1113521 & Lung & 0 & 0 & 0 \\
\hline 15P6219928-1 & Lung & 13 & 0.059923077 & 0.070504555 \\
\hline 15P6653082-1 & Lung & 5 & 0.1208 & 0.108767513 \\
\hline 15P6652808-1 & Lung & 1 & 0.006 & 0 \\
\hline 15P6652311-1 & Lung & 0 & 0 & 0 \\
\hline 15P0201548-1 & Lung & 9 & 0.048666667 & 0.056340384 \\
\hline 15P6653173-1 & Lung & 7 & 0.181 & 0.17344066 \\
\hline 14P1112646 & Lung & 0 & 0 & 0 \\
\hline 15P6651204-1 & Lung & 17 & 0.110705882 & 0.088645439 \\
\hline 14P1114306-1 & Lung & 3 & 0.027333333 & 0.161816718 \\
\hline 15P1825167-1 & Lung & 7 & 0.059714286 & 0.073946443 \\
\hline 15P6658740-1 & Lung & 0 & 0 & 0.110454377 \\
\hline
\end{tabular}




\begin{tabular}{|c|c|c|c|c|}
\hline 15P6215168-1 & Lung & 11 & 0.093636364 & 0.096164398 \\
\hline 15P0201565-1 & Lung & 0 & 0 & 0 \\
\hline 14P1012082 & Lung & 1 & 0.01 & 0 \\
\hline 15P6226247-1 & Lung & 2 & 0.0175 & 0.280374985 \\
\hline 15P6653260-1 & Lung & 5 & 0.206 & 0.303675861 \\
\hline 15P6653227-1 & Lung & 3 & 0.068 & 0.299663748 \\
\hline 15P6220091-1 & Lung & 11 & 0.137181818 & 0.081197413 \\
\hline 15P0047208-1 & Lung & 6 & 0.2663333333 & 0.3484067 \\
\hline 15P6653258-1 & Lung & 3 & 0.009 & 0 \\
\hline 15P6651928-1 & Lung & 18 & 0.226111111 & 0.266998073 \\
\hline 15P6652785-1 & Lung & 7 & 0.086142857 & 0.107690034 \\
\hline 15P6652362-1 & Lung & 1 & 0.143 & 0.154604273 \\
\hline 15P6650491-1 & Lung & 13 & 0.148615385 & 0.235123776 \\
\hline 14P1113520 & Lung & 23 & 0.169956522 & 0.123868105 \\
\hline 15P6219987-1 & Lung & 1 & 0.01 & 0 \\
\hline 14P1007586-1 & Lung & 0 & 0 & 0 \\
\hline 14P1113755-1 & Lung & 0 & 0 & 0 \\
\hline 14P1114095-1 & Lung & 0 & 0 & 0 \\
\hline 15P6652480-1 & Lung & 0 & 0 & 0 \\
\hline 15P6652783 & Lung & 0 & 0 & 0 \\
\hline 15P6653171-1 & Lung & 3 & 0.007 & 0 \\
\hline 15P6653198-1 & Lung & 0 & 0 & 0 \\
\hline 15P6653226-1 & Lung & 0 & 0 & 0 \\
\hline 15P6653230-1 & Lung & 2 & 0.0665 & 0.016422112 \\
\hline 14P1012513 & Lung & 10 & 0.0702 & 0.078008495 \\
\hline 15P6658643-1 & Lung & 2 & 0.0085 & 0 \\
\hline 15P6658528-1 & Lung & 2 & 0.0105 & 0 \\
\hline 15P6652977-1 & Lung & 9 & 0.071 & 0.307768202 \\
\hline 15P6652427-1 & Lung & 2 & 0.0165 & 0 \\
\hline 15P6653237-1 & Lung & 0 & 0 & 0 \\
\hline 14P1114105-1 & Lung & 1 & 0.018 & 0.02133771 \\
\hline 15P6656800-1 & Lung & 1 & 0.01 & 0 \\
\hline 15P6658628-1 & Lung & 3 & 0.027333333 & 0.020970937 \\
\hline 15P6651917-1 & Lung & 5 & 0.0854 & 0.127497145 \\
\hline 14P1112647-1 & Lung & 0 & 0 & 0 \\
\hline 14P1113491 & Lung & 1 & 0.005 & 0 \\
\hline 14P1007923 & Lung & 0 & 0 & 0 \\
\hline 15P6651916-1 & Lung & 0 & 0 & 0 \\
\hline 15P6653225-1 & Lung & 3 & 0.0326666667 & 0.045263244 \\
\hline 15P6651205-1 & Lung & 1 & 0.075 & 0 \\
\hline 14P0891664 & Lung & 6 & 0.169333333 & 0 \\
\hline 15P6653385-1 & Lung & 1 & 0.014 & 0 \\
\hline 15P6652980-1 & Lung & 2 & 0.058 & 0.03019133 \\
\hline 15P6653250-1 & Lung & 7 & 0.032285714 & 0.027567631 \\
\hline
\end{tabular}




\begin{tabular}{|c|c|c|c|c|}
\hline 15P6658739-1 & Lung & 1 & 0.027 & 0.027148833 \\
\hline 15P6653239-1 & Lung & 0 & 0 & 0 \\
\hline 14P1005328-1 & Lung & 0 & 0 & 0 \\
\hline 15P0201529-1 & Lung & 1 & 0.005 & 0 \\
\hline 15P0201544-1 & Lung & 0 & 0 & 0 \\
\hline 15P6653264-1 & Lung & 2 & 0.01 & 0 \\
\hline 14P0973622 & Lung & 2 & 0.0115 & 0 \\
\hline 14P1011995 & Lung & 0 & 0 & 0 \\
\hline 15P6651144-1 & Lung & 2 & 0.025 & 0.033178599 \\
\hline 15P6653235-1 & Lung & 9 & 0.047666667 & 0.033770392 \\
\hline 14P1114760-1 & Lung & 0 & 0 & 0 \\
\hline 15P6653208-1 & Lung & 0 & 0 & 0 \\
\hline 15P6651315-4 & Lung & 1 & 0.01 & 0 \\
\hline 15P6653190-1 & Lung & 12 & 0.057416667 & 0.055379965 \\
\hline 15P6653196-1 & Lung & 0 & 0 & 0.030212536 \\
\hline 15P6653212-1 & Lung & 2 & 0.032 & 0.042098043 \\
\hline 15P6658738-1 & Lung & 1 & 0.045 & 0.037710971 \\
\hline 15P6220092-1 & Lung & 8 & 0.04775 & 0.049817947 \\
\hline 14P1114307-1 & Lung & 0 & 0 & 0 \\
\hline 14P1007455 & Lung & 0 & 0 & 0 \\
\hline 15P6652773-1 & Lung & 4 & 0.0575 & 0.02997527 \\
\hline 14P1113496 & Lung & 21 & 0.044619048 & 0.048638729 \\
\hline 15P6653224-1 & Lung & 1 & 0.006 & 0 \\
\hline 15P6652486-1 & Lung & 1 & 0.032 & 0.044580806 \\
\hline 14P1113495 & Lung & 1 & 0.008 & 0 \\
\hline 15P6219936-1 & Lung & 0 & 0 & 0 \\
\hline 15P6654813-1 & Unknow & 1 & 0.005 & 0 \\
\hline 15P6653144-1 & Liver & 5 & 0.0124 & 0 \\
\hline 14P1009599 & Liver & 0 & 0 & 0 \\
\hline 15P6653216-1 & Liver & 8 & 0.146125 & 0.196204318 \\
\hline 15P6658494-1 & Liver & 3 & 0.088666667 & 0.092800381 \\
\hline 14P1114322-1 & Liver & 4 & 0.23925 & 0.372007165 \\
\hline 14P1111984 & Liver & 0 & 0 & 0.046137791 \\
\hline 15P6653207-1 & Liver & 0 & 0 & 0 \\
\hline 15P6653254-1 & Liver & 0 & 0 & 0 \\
\hline 15P6653229-1 & Liver & 3 & 0.027666667 & 0.03388115 \\
\hline 15P6657920-1 & Cervical & 1 & 0.01 & 0 \\
\hline 15P6215252-1 & Cervical & 2 & 0.2015 & 0 \\
\hline 15P6652285-1 & Cervical & 6 & 0.040166667 & 0.047674679 \\
\hline 14P1114040-1 & Cervical & 0 & 0 & 0 \\
\hline 15P6226129-1 & Cervical & 0 & 0 & 0 \\
\hline 14P1115157-1 & Cervical & 10 & 0.0371 & 0.048833597 \\
\hline 15P6219937-1 & Cervical & 11 & 0.034090909 & 0.061155945 \\
\hline 15P6658736-1 & Cervical & 6 & 0.015166667 & 0.019174428 \\
\hline
\end{tabular}




\begin{tabular}{|c|c|c|c|c|}
\hline 14P1012070 & Bone & 3 & 0.011333333 & 0 \\
\hline 14P1112476 & Bone & 0 & 0 & 0 \\
\hline 14P1012073 & Bone & 0 & 0 & 0 \\
\hline 15P6652525-1 & Bone & 0 & 0 & 0.050822655 \\
\hline 15P6651124-1 & Melanoma & 7 & 0.038857143 & 0.05140447 \\
\hline 15P6653142-1 & Synovial_sarcoma & 0 & 0 & 0 \\
\hline 15P6652777-1 & Glioblastoma & 0 & 0 & 0 \\
\hline 14P1113000 & Thyroid & 0 & 0 & 0 \\
\hline 14P1113002 & Colorectal & 0 & 0 & 0 \\
\hline 14P1114106-1 & Colorectal & 0 & 0 & 0 \\
\hline 15P6658485-1 & Colorectal & 0 & 0 & 0.269176289 \\
\hline 15P6658716-1 & Colorectal & 11 & 0.259 & 0.174174302 \\
\hline 15P6653204-1 & Colorectal & 5 & 0.3136 & 0.201413403 \\
\hline 15P6515536R-1 & Colorectal & 0 & 0 & 0 \\
\hline 14P1007519 & Colorectal & 11 & 0.210727273 & 0.211610161 \\
\hline 15P0201540-1 & Colorectal & 3 & 0.047333333 & 0.371726724 \\
\hline 15P6653221-1 & Colorectal & 1 & 0.006 & 0 \\
\hline 14P1006572 & Colorectal & 3 & 0.0436666667 & 0.051791102 \\
\hline 14P0975261 & Lymphoma & 1 & 0.009 & 0 \\
\hline 15P6653186-1 & Lymphoma & 5 & 0.1298 & 0.061800504 \\
\hline 15P6655982-1 & Lymphoma & 36 & 0.191555556 & 0.27810369 \\
\hline 15P6652491-1 & Ovarian & 0 & 0 & 0 \\
\hline 15P6650887-1 & Ovarian & 1 & 0.068 & 0 \\
\hline 15P6654500-1 & Ovarian & 0 & 0 & 0 \\
\hline 15P6656825-1 & Ovarian & 2 & 0.02 & 0 \\
\hline 15P6651892-1 & Ovarian & 0 & 0 & 0 \\
\hline 15P6652359-1 & Ovarian & 4 & 0.2075 & 0.227239234 \\
\hline 14P1004412-1 & Ovarian & 14 & 0.099357143 & 0.223643992 \\
\hline 15P6653234-1 & Ovarian & 6 & 0.193166667 & 0.247161438 \\
\hline 15P6653170-1 & Ovarian & 11 & 0.011818182 & 0 \\
\hline 15P6652786-1 & Ovarian & 3 & 0.016 & 0 \\
\hline 14P0973640 & Ovarian & 1 & 0.008 & 0 \\
\hline 15P6656801-1 & Ovarian & 1 & 0.007 & 0 \\
\hline 15P6653192-1 & Ovarian & 4 & 0.08775 & 0.053035986 \\
\hline 15P6653240-1 & Ovarian & 2 & 0.005 & 0 \\
\hline 15P6653220-1 & Ovarian & 1 & 0.005 & 0 \\
\hline 15P6658589-1 & Urinary & 15 & 0.420133333 & 0.629339452 \\
\hline 15P6652774-1 & Urinary & 10 & 0.0404 & 0.05919783 \\
\hline 15P6653011-1 & Brain & 1 & 0.014 & 0 \\
\hline $15 S 6651920-1$ & Prostate & 6 & 0.0346666667 & 0.086907028 \\
\hline 15P6653252-1 & Prostate & 3 & 0.1156666667 & 0.090258107 \\
\hline 15P6651145-1 & Prostate & 5 & 0.223 & 0.253668689 \\
\hline 15P6652775-1 & Prostate & 5 & 0.2786 & 0.615161866 \\
\hline 15P6653178-1 & Prostate & 8 & 0.136625 & 0.212796787 \\
\hline
\end{tabular}




\begin{tabular}{|c|c|c|c|c|}
\hline 15P6653248-1 & Prostate & 2 & 0.0065 & 0.033942454 \\
\hline 15P6653222-1 & Prostate & 6 & 0.015666667 & 0.028462785 \\
\hline 15P6656542-1 & Sarcoma & 2 & 0.2815 & 0.102729947 \\
\hline 14P1113655 & Sarcoma & 0 & 0 & 0 \\
\hline 15P6226230-1 & Breast & 0 & 0 & 0 \\
\hline 15P6652157-1 & Breast & 5 & 0.0378 & 0.051497365 \\
\hline 15P6651349-1 & Breast & 3 & 0.076333333 & 0.109239902 \\
\hline 15P6653217-1 & Breast & 0 & 0 & 0 \\
\hline 15P6653201-1 & Breast & 6 & 0.122833333 & 0.069904242 \\
\hline 14P0973390 & Breast & 3 & 0.179666667 & 0.229327757 \\
\hline 15P6653206-1 & Breast & 2 & 0.183 & 0.339381076 \\
\hline 14P0951863 & Breast & 26 & 0.098884615 & 0.510480856 \\
\hline 15P6220005-1 & Breast & 1 & 0.005 & 0 \\
\hline 14P1005818-1 & Breast & 2 & 0.101 & 0.260254934 \\
\hline 14P1005818-2 & Breast & 2 & 0.1915 & 0.475762298 \\
\hline 15P6653193-1 & Breast & 6 & 0.024333333 & 0.030172081 \\
\hline 15P6651130-1 & Breast & 0 & 0 & 0 \\
\hline 15P0201525-1 & Breast & 0 & 0 & 0 \\
\hline 14P1113686 & Breast & 3 & 0.013666667 & 0 \\
\hline 15P6653218-1 & Breast & 4 & 0.2715 & 0.272666702 \\
\hline 15P6658732-1 & Kidney & 0 & 0 & 0 \\
\hline 15P6653189-1 & Kidney & 10 & 0.116 & 0.139971233 \\
\hline 15P6651925-1 & Kidney & 0 & 0 & 0 \\
\hline 15P6653185-1 & Kidney & 24 & 0.034541667 & 0.043863828 \\
\hline 14P1007957-1 & Esophagus & 0 & 0 & 0 \\
\hline 15P6658733-1 & Esophagus & 0 & 0 & 0 \\
\hline 14P1010179 & Esophagus & 13 & 0.091692308 & 0.148532209 \\
\hline 14P1112999 & Esophagus & 3 & 0.015666667 & 0.037563001 \\
\hline 15P6652787-1 & Odiduct & 5 & 0.138 & 0.088576241 \\
\hline 15P6653228-1 & Head\&Neck & 0 & 0 & 0 \\
\hline 15P6652981-1 & Head\&Neck & 5 & 0.0334 & 0.036663166 \\
\hline 15P6654180-1 & Head\&Neck & 6 & 0.14 & 0.343960522 \\
\hline 15P6653191-1 & Head\&Neck & 3 & 0.086333333 & 0.13298039 \\
\hline 14P1114363-1 & Unknow & 15 & 0.1008 & 0.170220157 \\
\hline 14P1009600 & Unknow & 10 & 0.2589 & 0.221831563 \\
\hline 14P1011058 & Unknow & 2 & 0.0095 & 0 \\
\hline 14P1114098-1 & Unknow & 7 & 0.029142857 & 0 \\
\hline 14P1114373-1 & Unknow & 1 & 0.017 & 0 \\
\hline 15P6651951-1 & Stomach & 0 & 0 & 0 \\
\hline 15P6652784-1 & Stomach & 52 & 0.144423077 & 0.294842894 \\
\hline 15P6653261-1 & Stomach & 0 & 0 & 0 \\
\hline 15P6653197-1 & Stomach & 0 & 0 & 0 \\
\hline 15P6653205-1 & Stomach & 0 & 0 & 0 \\
\hline 14P1012171 & Stomach & 0 & 0 & 0.04230244 \\
\hline
\end{tabular}




$\begin{array}{llrrr}\text { 14P1006996-1 } & \text { Stomach } & 10 & 0.1993 & 0.378794082 \\ \text { 15P6653172-1 } & \text { Stomach } & 10 & 0.0269 & 0 \\ 14 \mathrm{P} 1008017-1 & \text { Stomach } & 0 & 0 & 0 \\ 15 \mathrm{P} 6653195-1 & \text { Stomach } & 8 & 0.0365 & 0.063398046 \\ 14 \mathrm{P} 1007958-1 & \text { Stomach } & 0 & 0 & 0.042912187 \\ \text { 15P1875721-1 } & \text { GIST } & 0 & 0 & 0 \\ \text { 15P6651691-1 } & \text { Unknow } & 1 & 0.009 & 0.050555753 \\ \text { 15P6653200-1 } & \text { Fibrosarcoma } & 6 & 0.062166667 & 0.091158194 \\ \text { 15P6653194-1 } & \text { SIST } & 0 & 0 & 0 \\ \text { 15P6653187-1 } & \text { Thymic } & 6 & 0.275 & 0.26576364 \\ \text { 14P1113517 } & \text { Pancrease } & 1 & 0.006 & 0 \\ \text { 15P6653979-1 } & \text { Pancrease } & 2 & 0.1355 & 0.095874427 \\ \text { 15P6652481-1 } & \text { Pancrease } & 0 & 0 & 0 \\ \text { 15P6658045-1 } & \text { Pancrease } & 8 & 0.336625 & 0.322894514 \\ \text { 15P6653247-1 } & \text { Pancrease } & 13 & 0.011692308 & 0 \\ \text { 14P1112533 } & \text { Pancrease } & 0 & 0 & 0 \\ \text { 14P1011866 } & \text { Pancrease } & 2 & 0.0115 & 0 \\ \text { 15P6653249-1 } & \text { Pancrease } & 1 & 0.1 & 0.138650611 \\ \text { 15P6226233-1 } & \text { Pancrease } & 7 & 0.037242546 \\ \text { 15P6653259-1 } & \text { Pancrease } & 4 & 0.0405 & 0.028335921 \\ \text { 15P6226237-1 } & \text { Pancrease } & 5 & 0.1994 & 0.156404556 \\ \text { 15P6650388-1 } & \text { Pancrease } & 1 & 0.016 & 0 \\ \text { 15P6653210-1 } & \text { Endometrium } & 20 & 0.075 & 0 \\ \text { 14P1112320 } & \text { Trophoblastic } & 1 & & 0\end{array}$

